\title{
VECTORIAL CRYSTALLIZATION PROBLEMS AND COLLECTIVE BEHAVIOR
}

\author{
L. DE LUCA, A. NINNO, AND M. PONSIGLIONE
}

\begin{abstract}
We propose and analyze a class of vectorial crystallization problems, with applications to crystallization of anisotropic molecules and collective behavior such as birds flocking and fish schooling.

We focus on two-dimensional systems of "oriented" particles: Admissible configurations are represented by vectorial empirical measures with density in $\mathcal{S}^{1}$. We endow such configurations with a graph structure, where the bonds represent the "convenient" interactions between particles, and the proposed variational principle consists in maximizing their number. The class of bonds is determined by hard sphere type pairwise potentials, depending both on the distance between the particles and on the angles between the segment joining two particles and their orientations, through threshold criteria.

Different ground states emerge by tuning the angular dependence in the potential, mimicking ducklings swimming in a row formation and predicting as well, for some specific values of the angular parameter, the so-called diamond formation in fish schooling.
\end{abstract}

Keywords: Crystallization; Collective behavior; Graph theory; Variational METHODS.

AMS SUBJECT ClASSificATiOns: 70C20, 05C10, 49J45, 82D25.

\section{Contents}

Introduction

1. Preliminaries on planar graphs 3

2. The variational model 5

2.1. Vectorial empirical configurations and their energy 6

2.2. Discrete graph representation 6

2.3. Basic qualitative properties of the ground states of $\mathcal{E}^{\gamma} \quad 7$

3. Rhombic ground states for $\gamma=\frac{\sqrt{3}}{2} \quad 10$

3.1. Geometric properties of admissible configurations 11

3.2. Geometric decomposition of the energy 12

3.3. Diamond formations 14

3.4. Compactness of quasi-minimizers 16

Appendix A. Auxiliary lemmas 20

References $\quad 28$

\section{INTRODUCTION}

The crystallization problem consists in understanding periodic configurations of atoms or molecules; self organization in ordered configurations is a general issue in nature and a central 
problem in biology [18]: birds flocking and fish schooling are typical examples of collective behavior and formation of ordered structures $[19,11]$.

Variational principles are transversal in nature, and in view of their predictive properties and ability to synthesize modeling perspectives, they have been a sound and fruitful reading key of many complex phenomena; relevant configurations of particles in ordered structures can be detected as ground states of energy functionals: Classical potentials adopted in the variational formulations involve pairwise interactions depending on the mutual distances between the particles; among them, short range repulsive/long range attractive potentials are very relevant, predicting in particular regular triangular lattices [20] (see also [5] for a crystallization result in the square lattice).

In this paper, we consider systems composed by elements, referred to as particles, endowed with an orientation that affects their interactions, and in turn the specific ground states. This is the case of anisotropic, e.g., elongated molecules (as proteins often are), where the orientation is given by the shape of the molecule, as well as the case of collective behaviors, where each individual is oriented according with its visual cone, or (mostly equivalently) with its velocity. Such orientations may induce highly anisotropic structures: Patchy particles with tetrahedral symmetry form diamond lattices [17] and diamond formations turn out to be energetically efficient for swimming of schooled fishes [6,21, 15]; ducklings move in a row formation, while the so called $V$-formation is convenient for ducks as well as for many other birds migration [11].

Although this kind of interactions, depending on mutual positions and orientations, attracted much attention in modeling and simulation issues, their mathematical analysis received until now only occasional interest. Also the theory of boids [19], developed to replicate birds flight, while having a big impact also for entertainment purposes, to the best of our knowledge, still lacks of a rigorous theoretical analysis.

The purpose of this paper is to provide a solid mathematical ground to describe and attack this kind of problems, and to propose and analyze in details a basic simple model in two dimensions able to predict diamond formations. The model is based on classical variational approaches to crystallization problems, but dealing with vectorial (rather than scalar) empirical measures with densities on the unit circle $\mathcal{S}^{1}$, taking into account the orientation of the particles. The optimal configuration is assumed to maximize the number of convenient interactions between particles, where the notion of convenience is determined by the mutual position and orientation between pairs of particles. Loosely speaking, our model is based on representing the admissible configurations as graphs, where the bonds are determined by the vectorial empirical measures, while the variational principle consists just in maximizing the number of such (convenient) bonds. A relevant issue of the proposed model is that, in defining the graph structure, and, in turn, the class of bonds, the positional and orientational variables are coupled; in this respect our model is also able to predict, rather than assume, alignment of orientations. Clearly, the specific rules determining the favorable interactions should rely on specific modeling considerations: for instance, in a pair of caudal swimmers the energy spent by the follower depends on the vortices in the fluid produced by the leader, and hence its energetically saving position depends on the angle between the direction of swimming and that of the line joining the two swimmers $[11,7]$. The terrific simplification we introduce in these modeling issues consists in assuming that both positional and orientational variables determine the class of convenient interactions only through threshold criteria, tuned by only two parameters, one acting on the mutual positions and the other one on both mutual positions and orientations. The positional short range repulsive/long range attractive behavior 
is mimicked by the hard sphere formalism as in the Heitmann-Radin model [13] (see also $[3,9,10,14,12])$ : particles are represented as discs with fixed radius that cannot overlap and that may interact only if they are tangent each other. Such interaction is switched on only if the angle formed by the orientations of both particles and the segment joining them is smaller than a given angular parameter, acting as a threshold. Therefore, renormalizing the hard disc radius, the model depends only on the angular parameter representing the amplitude of the visual cone of each particle.

Varying such a parameter, we find different ground states (see Subsection 2.3). Clearly, the 0-angular visual cone enforces one-dimensional ground states where particles have all the same orientation and are aligned on a row oriented accordingly, mimicking cyclist and duckling formations. On the other hand, tuning off the dependence on the orientation variable, the proposed model gives back the Heitmann-Radin model and the corresponding regular triangular lattice. Remarkably, for a specific choice of the angular visual cone, we show that diamond configurations minimize the energy; furthermore, in the thermodynamic limit as the number of particles diverges we show that all configurations with suitable energy bounds from above, consist in patched configurations of diamond formations with bounded perimeter.

While the specific model analyzed in details is very basic, the proposed methodology seems to be robust and could be enriched and generalized in many directions. As for instance, one could relax the threshold criteria seeking for other relevant configurations, like the Vformation. Further restrictions of our model are the two-dimensional setting and the fact that long range interactions are neglected. Moreover, our purely variational and static approach could be compared with reacher models [4] accounting for topological rather than metric distances, fluctuations and dynamical aspects. In particular, we remark that the orientational variable in our model has only a purely ideal interpretation in terms of the velocity of the particles; a justification of such an interpretation and rigorous connections with true dynamical models could deserve further investigation.

The paper is organized as follows. In Section 1 we collect some preliminaries on planar graphs that will be used throughout the paper. Section 2 is devoted to the description of our model and to the analysis of the qualitative properties of the ground states. In Section 3 we prove the minimality of the diamond formation and the compactness properties of quasiminimizers of the energy. Finally, some technical lemmas and their proofs are collected in Appendix A.

\section{Preliminaries on Planar graphS}

Here we collect some notions and notation on planar graphs that will be adopted in this paper.

Let $X$ be a finite subset of $\mathbb{R}^{2}$ and let $E d$ be a given subset of $E(X)$, where

$$
\mathrm{E}(\mathrm{X}):=\left\{\{x, y\} \subset \mathbb{R}^{2}: x, y \in \mathrm{X}, x \neq y\right\} .
$$

The pair $\mathrm{G}=(\mathrm{X}, \mathrm{Ed})$ is called graph; $\mathrm{X}$ is called the set of vertices of $\mathrm{G}$ and $\mathrm{Ed}$ is called the set of edges (or bonds) of $\mathrm{G}$.

Given $\mathrm{X}^{\prime} \subset \mathrm{X}$ we denote by $\mathrm{G}_{\mathbf{X}^{\prime}}$ the subgraph (or restriction) of $\mathrm{G}$ generated by $\mathrm{X}^{\prime}$, defined by $\mathrm{G}_{\mathbf{X}^{\prime}}=\left(\mathrm{X}^{\prime}, \mathrm{Ed}^{\prime}\right)$ where $\mathrm{Ed}^{\prime}:=\left\{\left\{x^{\prime}, y^{\prime}\right\} \in \mathrm{Ed}: x^{\prime}, y^{\prime} \in \mathrm{X}^{\prime}\right\}$.

Definition 1.1. We say that two points $x, z \in \mathrm{X}$ are connected and we write $x \sim z$ if there exist $M \in \mathbb{N}$ and a path $x=y_{0}, \ldots, y_{M}=z$ such that $\left\{y_{m-1}, y_{m}\right\} \in \mathrm{Ed}$ for every $m=1, \ldots, M-1$. We say that $\mathrm{G}_{\mathrm{X}_{1}}, \ldots, \mathrm{G}_{\mathrm{X}_{K}}$ with $K \in \mathbb{N}$ are the connected components of 
$\mathrm{G}$ if $\left\{\mathrm{X}_{1}, \ldots, \mathrm{X}_{K}\right\}$ is a partition of $\mathrm{X}$ and for every $k, k^{\prime} \in\{1, \ldots, K\}$ with $k \neq k^{\prime}$ it holds

$$
\begin{array}{cl}
x_{k} \sim y_{k} & \text { for every } x_{k}, y_{k} \in \mathrm{X}_{k}, \\
x_{k} \nsim x_{k^{\prime}} & \text { for every } x_{k} \in \mathrm{X}_{k}, x_{k^{\prime}} \in \mathrm{X}_{k^{\prime}} .
\end{array}
$$

If $\mathrm{G}$ has only one connected component we say that $\mathrm{G}$ is connected.

We say that $\mathrm{G}$ is planar if for every pair of (distinct) bonds $\left\{x_{1}, x_{2}\right\},\left\{y_{1}, y_{2}\right\} \in \mathrm{Ed}$, the (open) segments $\left(x_{1}, x_{2}\right)$ and $\left(y_{1}, y_{2}\right)$ have empty intersection.

From now on we assume that $\mathrm{G}=(\mathrm{X}, \mathrm{Ed})$ is planar, so that we can introduce the notion of face (see also [9]).

By a face $f$ of $G$ we mean any open, bounded, connected component of $\mathbb{R}^{2} \backslash\left(X \cup \bigcup_{\{x, y\} \in \text { Ed }}[x, y]\right)$, which is also simply connected; here $[x, y]$ is the closed segment with extreme points $x$ and $y$. We denote by $\mathrm{F}^{\mathrm{sc}}(\mathrm{G})$, or simply by $\mathrm{F}(\mathrm{G})$, the set of faces of $\mathrm{G}$. Moreover, we denote by $\mathrm{F}^{\mathrm{nsc}}(\mathrm{G})$ the set of open, bounded, connected components of $\mathbb{R}^{2} \backslash\left(\mathrm{X} \cup \bigcup_{\{x, y\} \in \mathrm{Ed}}[x, y]\right)$, which are not simply connected. We warn the reader that, in standard literature, also the elements of $F^{\text {nsc }}(G)$ are called faces. Moreover we set

$$
O(\mathrm{G}):=\bigcup_{f \in \mathrm{F}(\mathrm{G})} \cos (f) .
$$

With a little abuse of language we will say that an edge $\{x, y\}$ lies on a set $E \subset \mathbb{R}^{2}$ if the segment $[x, y]$ is contained in $E$. We classify the edges in Ed in the following subclasses:

- $\mathrm{Ed}^{\mathrm{int}}$ is the set of interior edges, i.e., of edges lying on the boundary of two (distinct) faces;

- $\mathrm{Ed}^{\text {wire,ext }}$ is the set of exterior wire edges, i.e., of edges that do not lie on the boundary of any face;

- Ed ${ }^{\text {wire,int }}$ is the set of interior wire edges, i.e., of edges lying on the boundary of precisely one face but not on the boundary of its closure (or, equivalently, of $O(\mathrm{G})$ ) ;

- $\mathrm{Ed}^{\partial}$ is the set of boundary edges, i.e., of edges lying on $\partial O(\mathrm{G})$.

Analogously, for every face $f \in \mathrm{F}(\mathrm{G})$ one can define the following subclasses of edges delimiting $f$ :

- $E d^{\text {wire,int }}(f)$ is the set of edges lying on the boundary of $f$ but not on the boundary of the closure of $f$;

- $\mathrm{Ed}^{\partial}(f)$ is the set of edges lying on the boundary of the closure of $f$.

Finally, we define the Euler characteristic of the graph $G=(X, E d)$. To this purpose, set $l_{0}(\mathrm{G})=l_{0}^{\text {Euler }}(\mathrm{G}):=\sharp \mathrm{X}, l_{1}(\mathrm{G})=l_{1}^{\text {Euler }}(\mathrm{G}):=\sharp \mathrm{Ed}, l_{2}(\mathrm{G}):=\sharp \mathrm{F}(\mathrm{G})$, and $l_{2}^{\text {Euler }}(\mathrm{G}):=l_{2}(\mathrm{G})+$ $\sharp F^{\mathrm{nsc}}(\mathrm{G})$. Then, we introduce the standard Euler characteristic $\chi^{\text {Euler }}(\mathrm{G})$ of $G$, together with a slight variant $\chi(G)$ of it that will be useful for our purposes:

$$
\chi^{\text {Euler }}(\mathrm{G}):=\sum_{k=0}^{2}(-1)^{k} l_{k}^{\text {Euler }}(\mathrm{G}), \quad \chi(\mathrm{G}):=\sum_{k=0}^{2}(-1)^{k} l_{k}(\mathrm{G}) .
$$

In the next result we recall the classical Euler characteristic formula for planar graphs together with its analogous for $\chi(\mathrm{G})$.

Lemma 1.2. For every planar graph $\mathrm{G}$ the Euler characteristic $\chi^{\mathrm{Euler}}(\mathrm{G})$ is equal to the number of connected components of $\mathrm{G}$. Moreover

$$
\chi(\mathrm{G}) \geq 1,
$$


and the equality in (1.3) holds true whenever $\mathrm{G}$ is connected.

Proof. The first sentence in the statement is the classical Euler formula. As for the proof of formula (1.3), we observe that $\chi^{\text {Euler }}(\mathrm{G})-\chi(\mathrm{G})=\sharp \mathrm{F}^{\mathrm{nsc}}(\mathrm{G})$ and that for every $f \in \mathrm{F}^{\mathrm{nsc}}(\mathrm{G})$, each "hole" of $f$ "contains" at least a connected component of $\mathrm{G}$; by an easy induction argument on the number $\sharp F^{n s c}(G)$ of non simply connected faces one can get (1.3).

Finally, if $G$ is connected, then $\sharp F^{\mathrm{nsc}}(\mathrm{G})=0$ so that $\chi(\mathrm{G})=\chi^{\mathrm{Euler}}(\mathrm{G})=1$.

We define the graph-perimeter of $\mathrm{G}$ as

$$
\operatorname{Per}_{\mathrm{gr}}(\mathrm{G}):=\sharp \mathrm{Ed}^{\partial}+2 \sharp \mathrm{Ed}^{\text {wire,ext }} .
$$

Analogously, the graph-perimeter of a face $f$ is defined by

$$
\operatorname{Per}_{\mathrm{gr}}(f):=\sharp \mathrm{Ed}^{\partial}(f)+2 \sharp \mathrm{Ed}^{\text {wire, int }}(f) \text {. }
$$

Now we show how a planar graph can be triangulated, controlling the number of the required additional bonds. Since the triangulation procedure is local, we will focus on graphs having only one face.

Lemma 1.3. Let $\mathrm{G}=(\mathrm{X}, \mathrm{Ed})$ be a planar graph having only one face $f$. If $\operatorname{Per}_{\mathrm{gr}}(f) \geq 4$, then there exists a planar graph $\overline{\mathrm{G}}=(\mathrm{X}, \overline{\mathrm{E}} \mathrm{d})$ such that

(1) $\mathrm{Ed} \subset \overline{\mathrm{Ed}}$;

(2) all the faces of $\mathrm{F}(\overline{\mathrm{G}})$ are triangles;

(3) $(x, y) \subset f$ for every $\{x, y\} \in \mathrm{Ed} \backslash \mathrm{Ed}$;

(4) $\sharp \mathrm{Ed}=\sharp \mathrm{Ed}+\operatorname{Per}_{\mathrm{gr}}(f)-3$;

(5) $\sharp \mathrm{F}(\overline{\mathrm{G}})=\operatorname{Per}_{\mathrm{gr}}(f)-2$.

Proof. We proceed by induction on $\operatorname{Per}_{\mathrm{gr}}(f)$. If $\operatorname{Per}_{\mathrm{gr}}(f)=3,4$, the claims are clearly true. Assume now that $\operatorname{Per}_{\mathrm{gr}}(f) \geq 5$ and that the claims are satisfied for every graph whose only face $g$ satisfies $\operatorname{Per}_{\text {gr }}(g)<\operatorname{Per}_{\text {gr }}(f)$. We can always split $f$ into two simply connected sets $f_{1}, f_{2}$ by cutting $f$ with a segment $[x, y]$ joining two points $x, y \in \mathrm{X}$ such that $(x, y)$ is contained in $f$ (see for instance [16]). Setting $\mathrm{Ed}^{\prime}:=\mathrm{Ed} \cup\{x, y\}$, we have that the faces of $\mathrm{G}^{\prime}=\left(\mathrm{X}, \mathrm{Ed}^{\prime}\right)$ are exactly $f_{1}$ and $f_{2}$. Moreover, we have that

$$
\operatorname{Per}_{\mathrm{gr}}\left(f_{1}\right)+\operatorname{Per}_{\mathrm{gr}}\left(f_{2}\right)=\operatorname{Per}_{\mathrm{gr}}(f)+2 .
$$

As a consequence, $\operatorname{Per}_{\mathrm{gr}}\left(f_{i}\right)<\operatorname{Per}_{\mathrm{gr}}(f)$ for $i=1,2$. By induction, we can triangulate both $f_{1}$ and $f_{2}$ adding $\operatorname{Per}_{\mathrm{gr}}\left(f_{1}\right)-3$ and $\operatorname{Per}_{\mathrm{gr}}\left(f_{2}\right)-3$ edges respectively. In view of (1.6) this implies that the number of additional edges used to triangulate $f$ is

$$
1+\operatorname{Per}_{\mathrm{gr}}\left(f_{1}\right)-3+\operatorname{Per}_{\mathrm{gr}}\left(f_{2}\right)-3=\operatorname{Per}_{\mathrm{gr}}(f)-3 .
$$

Moreover, again by the inductive step and by (1.6) we have that

$$
\sharp \mathrm{F}(\overline{\mathrm{G}})=\operatorname{Per}_{\mathrm{gr}}\left(f_{1}\right)-2+\operatorname{Per}_{\mathrm{gr}}\left(f_{2}\right)-2=\operatorname{Per}_{\mathrm{gr}}(f)-2 .
$$

\section{The VARIATiOnal MODEL}

In this section we describe our model, introducing the energy functional, and we study qualitative properties of its minimizers and almost minimizers. 
2.1. Vectorial empirical configurations and their energy. Let $\mathcal{A C}$ be a given subset of

$$
\mathcal{C}:=\left\{(X, V): X=\left(x_{1}, \ldots, x_{N}\right) \in\left(\mathbb{R}^{2}\right)^{N}, V=\left(v_{1}, \ldots, v_{N}\right) \in\left(\mathcal{S}^{1}\right)^{N}, N \in \mathbb{N}\right\},
$$

where $\mathcal{S}^{1}$ denotes the set of unitary vectors of $\mathbb{R}^{2}$. Here and throughout the paper $\mathbb{N}$ denotes the set of positive integers. In the following we will refer to $\mathcal{A C}$ as the set of admissible (vectorial empirical) configurations.

For every $(X, V) \in \mathcal{C}$ with $X=\left(x_{1}, \ldots, x_{N}\right)$ we set $\mathrm{X}:=\left\{x_{1}, \ldots, x_{N}\right\}$; we will adopt such a notation also for a generic subset of $\mathbb{R}^{2}$ with $N$ elements. Moreover if $(X, V) \in \mathcal{A C} \mathcal{C}_{N}$, for every $x \in \mathrm{X}$ we denote by $v(x)$ the orientation associated to the point $x$, i.e., if $x=x_{i}$ for some $i=1, \ldots, N$, then $v(x)=v_{i}$.

We assume that there exists a given map Ed that at each element $(X, V)$ of $\mathcal{A C}$ associates a subset $\mathrm{Ed}(X, V)$ of $\mathrm{E}(\mathrm{X})$ (defined in (1.1)), referred to as the set edges (or bonds) of $(X, V)$.

We call energy any given functional $\mathcal{E}: \mathcal{A C} \rightarrow \mathbb{R}$ of the form $\mathcal{E}(X, V)=\mathscr{E}(X, V, \operatorname{Ed}(X, V))$; in this paper we consider energies of the following type

$$
\mathcal{E}(X, V):=-\sharp \operatorname{Ed}(X, V) .
$$

Such a choice of energy is very specific and of course it could be generalized in many perspectives; nevertheless, it leaves enough freedom in the choice of the criteria that determine the class $\mathcal{A C}$ of admissible configurations and the class of edges.

In this paper we focus on threshold criteria for defining both $\mathcal{A C}$ and Ed. We set

$$
\mathcal{A C}:=\{(X, V) \in \mathcal{C}:|x-y| \geq 1 \text { for every } x, y \in \mathrm{X} \text { with } x \neq y\} .
$$

Given $\gamma \in[0,1]$, for every $(X, V) \in \mathcal{C}$ with $X=\left(x_{1}, \ldots, x_{N}\right) \in\left(\mathbb{R}^{2}\right)^{N}$ and $V=\left(v_{1}, \ldots, v_{N}\right) \in$ $\left(\mathcal{S}^{1}\right)^{N}$ for some $N \in \mathbb{N}$ we define

$$
\begin{aligned}
\operatorname{Ed}^{\gamma}(X, V):= & \left\{\left\{x_{i}, x_{j}\right\} \in \mathrm{E}(\mathrm{X}):\left|x_{i}-x_{j}\right|=1,\right. \text { and } \\
& \text { either } \left.\left\langle x_{j}-x_{i}, v_{k}\right\rangle \geq \gamma \text { for } k=i, j \text { or }\left\langle x_{i}-x_{j}, v_{k}\right\rangle \geq \gamma \text { for } k=i, j\right\}
\end{aligned}
$$

and we set

$$
\mathcal{E}^{\gamma}(X, V):=-\sharp \mathrm{Ed}^{\gamma}(X, V) .
$$

In Section 3 we will focus on the case $\gamma=\frac{\sqrt{3}}{2}$; in Subsection 2.3 below, we briefly discuss what happens for different values of $\gamma$. The main purpose is to show the variety of ground states emerging from the only minimization of the basic energy $\mathcal{E}^{\gamma}$, depending on the choice of the edges in (2.2), i.e., on $\gamma$.

2.2. Discrete graph representation. Fix $\gamma \in[0,1]$. For every $(X, V) \in \mathcal{A C}$, we consider the graph $\mathrm{G}^{\gamma}(X, V)=\left(\mathrm{X}, \mathrm{Ed}^{\gamma}(X, V)\right)$, referred to as the bond graph of the configuration $(X, V)$. Since $(X, V) \in \mathcal{A C}$, we have that $\mathrm{G}^{\gamma}(X, V)$ is planar.

From now we will use the notions introduced in Section 1 for $\mathrm{G}=\mathrm{G}^{\gamma}(X, V)$ and $\mathrm{Ed}=$ $\operatorname{Ed}^{\gamma}(X, V)$. To ease the notation we set $\mathrm{F}^{\gamma}(X, V):=\mathrm{F}\left(\mathrm{G}^{\gamma}(X, V)\right), O^{\gamma}(X, V):=O\left(\mathrm{G}^{\gamma}(X, V)\right)$ and so on. Moreover, we denote by $\mathrm{Ed}^{\gamma, \partial}(X, V)$ the set of boundary edges of $\mathrm{G}^{\gamma}(X, V)$.

We define the perimeter $\operatorname{Per}^{\gamma}(X, V)$ of $\mathrm{G}^{\gamma}(X, V)$ as the perimeter of $O^{\gamma}(X, V)$, i.e.,

$$
\operatorname{Per}^{\gamma}(X, V):=\sharp \mathrm{Ed}^{\gamma, \partial}(X, V) .
$$

Notice that, since the edges have unitary length, $\operatorname{Per}^{\gamma}(X, V)$ is the standard perimeter of $O^{\gamma}(X, V)$, whereas $\operatorname{Per}_{\mathrm{gr}}^{\gamma}(X, V):=\operatorname{Per}_{\mathrm{gr}}\left(\mathrm{G}^{\gamma}(X, V)\right)$ represents the relaxation of $\operatorname{Per}^{\gamma}(X, V)$ with respect to outer approximations of $O^{\gamma}(X, V)$ with open sets. 
Definition 2.1. For every $(X, V) \in \mathcal{A C}$ we denote by $\partial^{\gamma} X$ the subset of $\mathrm{X}$ made by points which lie either on (at least) one boundary edge or on (at least) one exterior wire edge or on no edge. In the following, we will call interior points of $(X, V)$ the elements of $X \backslash \partial^{\gamma} X$.

2.3. Basic qualitative properties of the ground states of $\mathcal{E}^{\gamma}$. We first recall that the Heitmann-Radin energy defined in [13] is given by the number of pairs of tangent hard spheres. Such a model fits our formalism: the energy can be written as in (2.1) where the bonds are determined by the only hard sphere tangency condition, being independent of the orientations. More precisely, for every $(X, V) \in \mathcal{C}$ the Heitmann-Radin energy can be defined by

$$
\mathcal{E}_{H R}(X, V)=\left\{\begin{array}{ll}
-\sharp \mathrm{Ed}_{H R}(X) & \text { if }(X, V) \in \mathcal{A C} \\
+\infty & \text { otherwise }
\end{array},\right.
$$

with

$$
\operatorname{Ed}_{H R}(X):=\{\{x, y\} \in \mathrm{E}(\mathrm{X}):|x-y|=1\} .
$$

As proven in [13] (see also [9]), for every fixed $N \in \mathbb{N}$ the minimizers of the functional $\mathcal{E}_{H R}$ in

$$
\mathcal{A C}_{N}:=\left\{(X, V) \in \mathcal{A C} \cap\left(\left(\mathbb{R}^{2}\right)^{N} \times\left(\mathcal{S}^{1}\right)^{N}\right)\right\}
$$

are, up to a rotation and a translation, subsets of the unitary triangular lattice

$$
\mathcal{T}^{1}:=\left\{a(1 ; 0)+b\left(\frac{1}{2} ; \frac{\sqrt{3}}{2}\right): a, b \in \mathbb{Z}\right\} .
$$

For every $\gamma \in[0,1]$ and for every $(X, V) \in \mathcal{A C}$, we clearly have $\sharp \operatorname{Ed}^{\gamma}(X, V) \leq \sharp \mathrm{Ed}_{H R}(X)$ or, equivalently,

$$
\mathcal{E}^{\gamma}(X, V):=-\sharp \mathrm{Ed}^{\gamma}(X, V) \geq \sharp \mathcal{E}_{H R}(X, V) .
$$

Now, we briefly discuss some qualitative properties of the ground states and of almost minimizers of the energy $\mathcal{E}^{\gamma}$, for $\gamma$ varying in the range $[0,1]$. The significant case $\gamma=\frac{\sqrt{3}}{2}$ will be further analyzed in more details in Section 3.

The case $\gamma=0$. This case is a slight generalization of the Heitmann-Radin model, being the dependence on the orientations only fictitious. Indeed, for every given $N \in \mathbb{N}$ and $v \in \mathcal{S}^{1}$, let $\bar{V}:=\{v\}^{N}$ be a constant (arbitrarily chosen) orientation field. Then, for every $(X, V) \in \mathcal{A C}_{N}$, we have that $\mathrm{Ed}^{0}(X, \bar{V})=\operatorname{Ed}_{H R}(X)$, or, equivalently, $\mathcal{E}^{0}(X, \bar{V})=\mathcal{E}_{H R}(X, V)$. In particular, for configurations with constant orientation, the class of minimizers (resp. almost minimizers) of $\mathcal{E}^{0}$ coincides with the class of minimizers (resp. almost minimizers) of $\mathcal{E}_{H R}$, analyzed in $[13,9,8,3,10,12]$.

The case $0<\gamma \leq \frac{1}{2}$. In such a range, for suitably chosen constant orientations, the ground states are the same as in the Heitmann-Radin model; in particular, they are subsets of the unitary triangular lattice. Indeed, let $N \in \mathbb{N}$ and let $(X, V)$ be a minimizer of $\mathcal{E}_{H R}$ in $\mathcal{A C}_{N}$; by [13], up to a rigid motion, $\mathrm{X} \subset \mathcal{T}^{1}$. Therefore, setting $\bar{V}:=\{(1 ; 0)\}^{N}$, we have that $\operatorname{Ed}^{\gamma}(X, \bar{V})=\operatorname{Ed}_{H R}(X)$, which, in view of (2.6), yields the claim. Notice also that not all constant orientations do the job: Indeed, taking $(X, V)$ as above and $(X, \hat{V})$ with $\hat{V}:=\{(0 ; 1)\}^{N}$, we have that all the "horizontal bonds" in $\operatorname{Ed}_{H R}(X)$, namely, the bonds $\left\{x_{i}, x_{j}\right\}$ with $x_{j}-x_{i}=\lambda(1 ; 0)$ for some $\lambda \in\{-1,1\}$, do not belong to $\operatorname{Ed}^{\gamma}(X, \bar{V})$ and hence $\mathcal{E}^{\gamma}(X, \hat{V})>\mathcal{E}_{H R}(X, V)$ (see Figure 1$)$. 
A natural question is whether, in the range $\left(0, \frac{1}{2}\right]$, for every configuration $(X, V) \in \mathcal{A C}$ there exists a configuration $(X, \bar{V})$ (where $\bar{V}$ is a minimizer for fixed $X$ ) such that $\mathcal{E}^{\gamma}(X, \bar{V})=$ $\mathcal{E}_{H R}(X, V)$.
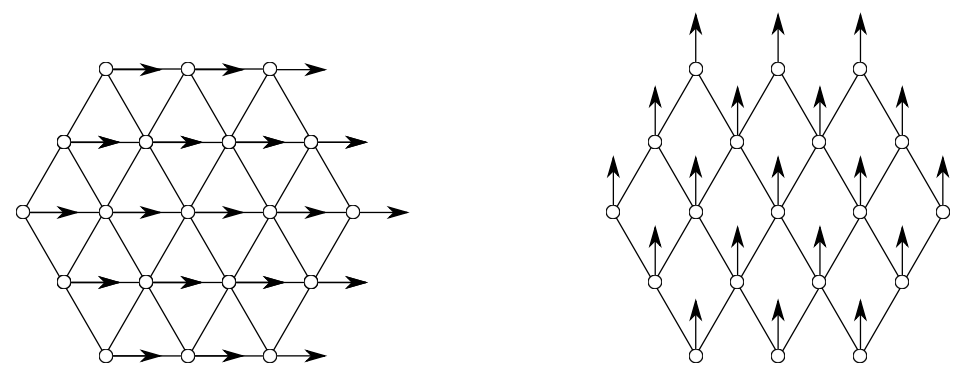

Figure 1. The unique, up to rotation, minimizer $X$ of the Heitmann-Radin energy for $N=19$. On the left: the configuration $(X, \bar{V})$. On the right: the configuration $(X, \hat{V})$.

The case $\frac{1}{2}<\gamma \leq \frac{\sqrt{3}}{2}$. In such a case we have that the maximal number of nearest neighbors of a point is equal to 4 . Indeed, let $(X, V) \in \mathcal{A C}$ and let $x \in \mathrm{X}$; without loss of generality we can assume that $x=0$ and that the orientation of $x$ is given by $(1 ; 0)$. Notice that if $y \in \mathbf{X}$ is such that $\{x, y\} \in \mathrm{Ed}^{\gamma}(X, V)$, then $y=(\cos \theta ; \sin \theta)$ for some $\theta \in\left(-\frac{\pi}{3}, \frac{\pi}{3}\right) \cup\left(\frac{2}{3} \pi, \frac{4}{3} \pi\right)$. By the very definition of $\mathcal{A C}$, we immediately have that there can be at most two nearest neighbors of $x$ of the type $y=(\cos \theta ; \sin \theta)$ with $\theta \in\left(-\frac{\pi}{3}, \frac{\pi}{3}\right)$ as well as with $\theta \in\left(\frac{2}{3} \pi, \frac{4}{3} \pi\right)$; in particular, the claim follows.

Now, we show that the asymptotic (as $N \rightarrow+\infty$ ) energy per particle for minimizers equals to -4 ; more precisely,

$$
-4 N \leq \min _{(X, V) \in \mathcal{A} \mathcal{C}_{N}} \mathcal{E}^{\gamma}(X, V) \leq-4 N+C N^{\frac{1}{2}},
$$

for some $C>0$ independent of $N$. Notice that the first inequality in (2.7) is a direct consequence of the fact that 4 is the maximal number of nearest neighbors; for what concerns the second inequality, for every $N \in \mathbb{N}$ let $V_{N}=\{(0 ; 1)\}^{N}$ and let $\mathrm{X}_{N}$ be a subset of $\mathcal{T}^{1}$ with cardinality equal to $N$ such that the graph $\mathrm{G}^{\gamma}\left(X_{N}, V_{N}\right)$ is connected, the elements of $\mathrm{F}^{\gamma}\left(X_{N}, V_{N}\right)$ are all unitary rhombuses, and $\sharp \partial^{\gamma} X_{N} \leq C N^{\frac{1}{2}}$ for some $C>0$ independent of $N$. Such configurations can be easily constructed (see, for instance, the configurations $\left(Y_{N}, W_{N}\right)$ provided by Definition 3.5 and Figure 3 below) and satisfy

$$
\mathcal{E}^{\gamma}\left(X_{N}, V_{N}\right) \leq-4 \sharp\left(X_{N} \backslash \partial X_{N}\right) \leq-4 N+4 C N^{\frac{1}{2}},
$$

from which the second inequality in (2.7) follows.

Notice also that small perturbations of the configuration $\left(X_{N}, V_{N}\right)$ constructed above still yields almost minimizers for $\mathcal{E}^{\gamma}$ satisfying (2.8). Indeed, for all $\rho>0$ set

$$
T^{\rho}:=\left(\begin{array}{ll}
1+\rho & 0 \\
0 & \frac{\sqrt{3-\rho^{2}-2 \rho}}{\sqrt{3}}
\end{array}\right),
$$


and notice that, for $\rho$ small enough, $T^{\rho}$ maps $\mathcal{T}^{1}$ into a unitary rhombic lattice. Now, for all $\rho>0$ let $X_{N}^{\rho}=\left(T^{\rho}\left(x_{1}\right), \ldots, T^{\rho}\left(x_{N}\right)\right)$, and notice that, for $\rho$ small enough, $\left(X_{N}^{\rho}, V_{N}\right) \in \mathcal{A C}_{N}$. Then, by an easy continuity argument, for all $\gamma \in\left(\frac{1}{2}, \frac{\sqrt{3}}{2}\right)$ there exists $\rho>0$ (depending on $\gamma$ ) such that $\mathcal{E}^{\gamma}\left(X_{N}^{\rho}, V_{N}\right)=\mathcal{E}^{\gamma}\left(X_{N}, V_{N}\right)$ and hence $\left(X_{N}^{\rho}, V_{N}\right)$ still satisfies (2.8). Analogously, one can easily see that energy is invariant also under small perturbations of the orientation field $V_{N}$. Finally, for $N=4$ one can check that the configurations described above provide all the minimizers of $\mathcal{E}^{\gamma}$.

The case $\frac{\sqrt{3}}{2}<\gamma<1$. We first show that in this case the maximal number of nearest neighbors of a point is equal to 2 . Indeed, let $(X, V) \in \mathcal{A C}$ and let $x \in \mathrm{X}$; without loss of generality we can assume that $x=0$ and that the orientation of $x$ is given by $(1 ; 0)$. Notice that if $y \in \mathrm{X}$ is such that $\{x, y\} \in \mathrm{Ed}^{\gamma}(X, V)$, then $y=(\cos \theta ; \sin \theta)$ for some $\theta \in\left(-\frac{\pi}{6}, \frac{\pi}{6}\right) \cup\left(\frac{5}{6} \pi, \frac{7}{6} \pi\right)$. By the very definition of $\mathcal{A C}$, we immediately have that there can be at most one nearest neighbor $y=(\cos \theta ; \sin \theta)$ of $x$ with $\theta \in\left(-\frac{\pi}{6}, \frac{\pi}{6}\right)$ as well as with $\theta \in\left(\frac{5}{6} \pi, \frac{7}{6} \pi\right)$; in particular, the claim follows.

For every $N \in \mathbb{N}$ we have

$$
-N \leq \min _{(X, V) \in \mathcal{A} \mathcal{C}_{N}} \mathcal{E}^{\gamma}(X, V) \leq-N+1
$$

where the second inequality follows by considering the competitor $(\bar{X}, \bar{V}) \in \mathcal{A C}_{N}$ with $\bar{X}=$ $((0 ; 0),(1 ; 0), \ldots,(N ; 0))$ and $\bar{V}=\{(1 ; 0)\}^{N}$.

For such a range of the parameter $\gamma$, we can characterize the minimizers of the energy $\mathcal{E}^{\gamma}$ for every $N \in \mathbb{N}$. To this purpose, we set $N_{\gamma}:=\left\lceil\frac{\pi}{\arccos \gamma}\right\rceil$, where $\lceil a\rceil:=\min \{m \in \mathbb{N} \cup\{0\}$ : $m \geq a\}$ for every $a>0$.

We first consider the case $N<N_{\gamma}$. Let $(X, V)$ be a minimizer of $\mathcal{E}^{\gamma}$ in $\mathcal{A C}_{N}$. We claim that, up to a relabeling, $\mathrm{X}=\left\{x_{1}, \ldots, x_{N}\right\}$ where $\left\{x_{j}, x_{k}\right\} \in \operatorname{Ed}^{\gamma}(X, V)$ if and only if $|i-j|=1$ and hence $\mathcal{E}^{\gamma}(X, V)=-N+1$. To prove the claim, assume by contradiction that there exist $3 \leq J \leq N$ and $y_{1}, \ldots, y_{J} \in \mathrm{X}$ such that $\left\{y_{j}, y_{j+1}\right\} \in \operatorname{Ed}^{\gamma}(X, V)$ for every $j=1, \ldots, J-1$, $\left\{y_{1}, y_{J}\right\} \in \operatorname{Ed}^{\gamma}(X, V)$, and $C:=\bigcup_{j=1}^{J-1}\left[y_{j}, y_{j+1}\right] \cup\left[y_{J}, y_{1}\right]$ is a simple and closed polygonal curve. Then, denoting by $\alpha_{j}$ the inner angles of the polygon enclosed by $C$ and setting $\alpha_{\bar{\jmath}}:=\min _{j \in\{1, \ldots, J\}} \alpha_{j}$, by Euler formula, we have

$$
J \alpha_{\bar{\jmath}} \leq \sum_{j=1}^{J} \alpha_{j}=(J-2) \pi,
$$

so that

$$
\pi-2 \arccos \gamma \leq \alpha_{\bar{\jmath}}<\left(1-\frac{2}{\frac{\pi}{\arccos \gamma}}\right) \pi,
$$

which yields the desired contradiction.

Now we consider the case $N \geq N_{\gamma}$. Let $\mathrm{X}$ be the set of the vertices of a regular unitary $N$-gon centered at the origin, and for every $x \in \mathrm{X}$ let $v(x)$ be the unitary vector orthogonal to $x$, with orientation chosen so that either $v(x) \times x \equiv+1$ or $v(x) \times x \equiv-1$ for all $x \in \mathrm{X}$. Since $N \geq N_{\gamma}$, it easily follows that $(X, V) \in \mathcal{A C}_{N}$; moreover, $\mathcal{E}^{\gamma}(X, V)=-N$, so that $(X, V)$ is a minimizer. We easily conclude that if $(X, V)$ is any minimizer of $\mathcal{E}^{\gamma}$ in $\mathcal{A C}_{N}$, then every $x \in \mathrm{X}$ has exactly two nearest neighbors. It follows that every minimizer $(X, V)$ of $\mathcal{E}^{\gamma}$ in $\mathcal{A C}_{N}$ satisfies the following properties: there exist $K \in \mathbb{N}$, a partition $\left\{\mathrm{X}_{1}, \ldots, \mathrm{X}_{K}\right\}$ of $\mathrm{X}$ and simple and closed curves $\Gamma_{1}, \ldots, \Gamma_{K}$ such that $\min _{k_{1} \neq k_{2}} \operatorname{dist}\left(\mathrm{X}_{k_{1}}, \mathrm{X}_{k_{2}}\right) \geq 1, \mathrm{X}_{k}$ is the set of nodes 
of $\Gamma_{k}$ and $\sharp \mathrm{X}_{k}=\mathcal{H}^{1}\left(\Gamma_{k}\right) \geq N_{\gamma}$ for every $k=1, \ldots, K$. Moreover, for every $n=1, \ldots, N$ the angles $\hat{x}_{n}$ at the node $x_{n}$ satisfy $\left|\hat{x}_{n}-\pi\right|<2 \arccos \gamma$. Viceversa, all the configurations above described, are admissible and have energy equal to $-N$, and hence provide all possible minimizers of $\mathcal{E}^{\gamma}$ in $\mathcal{A C}_{N}$.
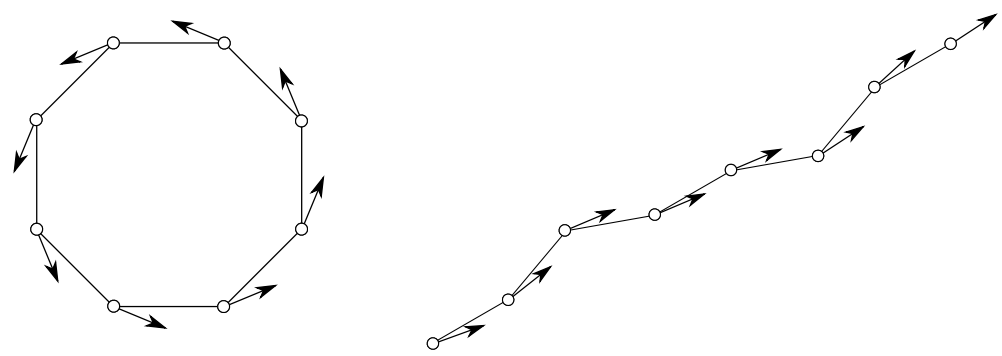

Figure 2. On the left: A minimizer for $N \geq N_{\gamma}$. On the right: A minimizer for $N<N_{\gamma}$.

The case $\gamma=1$. As for the range $\left(\frac{\sqrt{3}}{2}, 1\right)$, we have that also in this case the maximal number of nearest neighbors of a point is equal to 2. Moreover, given $(X, V) \in \mathcal{A C}$, we have that $\{x, y\} \in \operatorname{Ed}^{1}(X, V)$ if and only if $|x-y|=1$ and $v(x)=v(y)= \pm(x-y)$; it follows that the ground states of $\mathcal{E}^{1}$ in $\mathcal{A C}_{N}$ are made of $N$ aligned points forming a segment with constant tangent orientation, while the corresponding minimal energy is equal to $-N+1$.

Remark 2.2. In looking at minimal configurations, one could first fix $X$ and then minimize with respect to all possible orientation fields $V$, obtaining a reduced energy only depending on $\mathrm{X}$, and eventually further minimize with respect to $\mathrm{X}$. Notice also that further constraints could be enforced on $V$; for instance, denoting by $\bar{V}$ the average of the elements of $V$, one could plug some specific requirement on $|\bar{V}|$. Notice that $|\bar{V}|=1$ enforces constant orientation, while enforcing smallness conditions on $|\bar{V}|$ should favour cyclic-type configurations such as in the case $\gamma \in\left(\frac{\sqrt{3}}{2}, 1\right)$ and large $N$ considered above. Furthermore, in the energy functional a term penalizing variations of the orientation field could be added. One of the purposes of this paper is to show that, even in absence of such a penalization term, alignment of the orientation could be induced by the only minimization of $\mathcal{E}^{\gamma}$.

\section{Rhombic Ground states FOR $\gamma=\frac{\sqrt{3}}{2}$}

This section is devoted to the detailed analysis of minimizers and almost minimizers of the energy $\mathcal{E}^{\gamma}$ defined in $(2.3)$ for $\gamma=\frac{\sqrt{3}}{2}$. More precisely, we construct explicit rhombic minimizers (mimicking diamond formations) of the functional $\mathcal{E}^{\frac{\sqrt{3}}{2}}$ in $\mathcal{A C}_{N}$ for every $N \in \mathbb{N}$ and we obtain a compactness result for almost minimizers of $\mathcal{E}^{\frac{\sqrt{3}}{2}}$.

Since $\gamma$ is fixed to be equal to $\frac{\sqrt{3}}{2}$, in this section we omit the dependence on $\gamma$ in all the notations introduced above, i.e., for every $(X, V) \in \mathcal{A C}$ we set $\operatorname{Ed}(X, V):=\operatorname{Ed}^{\frac{\sqrt{3}}{2}}(X, V)$, $\mathcal{E}(X, V):=\mathcal{E}^{\frac{\sqrt{3}}{2}}(X, V), \mathrm{G}(X, V):=\mathrm{G}^{\frac{\sqrt{3}}{2}}(X, V), \partial X:=\partial^{\frac{\sqrt{3}}{2}} X$, and so on.

We start by proving some geometric properties of the bond graphs of the configurations in $\mathcal{A C}$. 
3.1. Geometric properties of admissible configurations. The following lemma provides geometric properties of the angles formed by two edges. Let $(X, V) \in \mathcal{A C}$. For every $x_{0}, x_{1}, x_{2} \in \mathrm{X}$ with $\left\{x_{0}, x_{1}\right\},\left\{x_{0}, x_{2}\right\} \in \mathrm{Ed}(X, V)$, the symbol $\widehat{x_{1} x_{0} x_{2}}$ - denotes the convex angle formed by the segments $\left[x_{0}, x_{1}\right]$ and $\left[x_{0}, x_{2}\right]$ whereas $\widehat{x_{1} x_{0} x_{2}}+$ denotes the concave one. Notice that $\widehat{x_{1} x_{0} x_{2}} \in(0, \pi], \widehat{x_{1} x_{0} x_{2}}+\in[\pi, 2 \pi)$ and

$$
\widehat{x_{1} x_{0} x_{2}}+\widehat{x_{1} x_{0} x_{2+}}=2 \pi \text {. }
$$

Lemma 3.1. Let $(X, V) \in \mathcal{A C}$. The following properties hold true.

(i) If $x_{0}, x_{1}, x_{2} \in \mathrm{X}$ are such that $\left\{x_{0}, x_{1}\right\},\left\{x_{0}, x_{2}\right\} \in \operatorname{Ed}(X, V)$, then

$$
\widehat{x_{1} x_{0} x_{2}} \in\left\{\frac{\pi}{3}\right\} \cup\left[\frac{2}{3} \pi, \pi\right], \quad \widehat{x_{1} x_{0} x_{2}} \in\left\{\frac{5}{3} \pi\right\} \cup\left[\pi, \frac{4}{3} \pi\right] .
$$

Moreover, if $\widehat{x_{1} x_{0} x_{2}}-=\frac{\pi}{3}$, then $v\left(x_{0}\right)$ is parallel to the bisector of $\widehat{x_{1} x_{0} x_{2}}-$ whereas, if $\widehat{x_{1} x_{0} x_{2}}=\frac{2}{3} \pi$, then $v\left(x_{0}\right)$ is orthogonal to the bisector of $\widehat{x_{1} x_{0} x_{2}}$.

(ii) If $x_{0}, x_{1}, x_{2}, x_{3} \in \mathrm{X}$ are such that $\left\{x_{0}, x_{1}\right\},\left\{x_{0}, x_{2}\right\},\left\{x_{0}, x_{3}\right\} \in \mathrm{Ed}(X, V)$, then

$$
\widehat{x_{1} x_{0} x_{2}}-\not \widehat{x_{2} x_{0} x_{3}} \text {. }
$$

(iii) Every $x \in \mathbf{X}$ can lie on at most four bonds. Moreover, in such a case, the four segments corresponding to the four bonds containing $x$ form equal opposite angles $\frac{\pi}{3}$ and $\frac{2}{3} \pi$.

Proof. We start by proving (3.1). Up to a rigid motion, we can assume that $x_{0}=(0 ; 0)=$ 0 and that $v\left(x_{0}\right)=(1 ; 0)=1$. By the very definition of $\operatorname{Ed}(X, V)$, we have that $x_{1}=$ $\left(\cos \vartheta_{1} ; \sin \vartheta_{1}\right)=e^{i \vartheta_{1}}$ and $x_{2}=\left(\cos \vartheta_{2} ; \sin \vartheta_{2}\right)=e^{i \vartheta_{2}}$ where $\vartheta_{j} \in\left[-\frac{\pi}{6}, \frac{\pi}{6}\right] \cup\left[\frac{5}{6} \pi, \frac{7}{6} \pi\right]$ and by the very definition of $\mathcal{A C}$ we get $\left|\vartheta_{1}-\vartheta_{2}\right| \geq \frac{\pi}{3}$. By combining the above conditions, (3.1) follows.

Now, if $\left|\vartheta_{1}-\vartheta_{2}\right|=\frac{\pi}{3}$, then $\left(\vartheta_{1} ; \vartheta_{2}\right)= \pm\left(-\frac{\pi}{6} ; \frac{\pi}{6}\right)$, which immediately implies that $v\left(x_{0}\right)$ is parallel to the bisector of the angle $\widehat{x_{1} x_{0} x_{2}}$. Analogously, the case $\left|\vartheta_{1}-\vartheta_{2}\right|=\frac{2}{3} \pi$ follows.

Now we prove (ii). Assume by contradiction that $\widehat{x_{1} x_{0} x_{2}}=\widehat{x_{2} x_{0} x_{3}}=: \alpha$. By (3.1), we have that either $\alpha=\frac{\pi}{3}$ or $\alpha=\frac{2}{3} \pi$; since the angles $\widehat{x_{1} x_{0} x_{2}}-\widehat{x_{2} x_{0} x_{3}}$ - are adjacent, by the last statement in (i), we get incompatible conditions on $v\left(x_{0}\right)$.

Finally, we prove (iii). We use the same argument of Subsection 2.3. Without loss of generality we can assume that $x=0$ and that $v(x)=(1 ; 0)=1$. Notice that if $y \in \mathbf{X}$ is such that $\{x, y\} \in \operatorname{Ed}(X, V)$, then $y=e^{i \vartheta}$ for some $\vartheta \in\left[-\frac{\pi}{6}, \frac{\pi}{6}\right] \cup\left[\frac{5}{6} \pi, \frac{7}{6} \pi\right]$. By the very definition of $\mathcal{A C}$, we immediately have that there can be at most two nearest neighbors of $x$ of the type $y=e^{i \vartheta}$ with $\vartheta \in\left[-\frac{\pi}{6}, \frac{\pi}{6}\right]$ and if there are exactly two nearest neighbors in such an interval they should be $x_{1}=e^{i \frac{\pi}{6}}$ and $x_{2}=e^{-i \frac{\pi}{6}}$. Similarly, there can be at most two nearest neighbors of $x$ for $\theta \in\left[\frac{5}{6} \pi, \frac{7}{6} \pi\right]$ and if there are exactly two nearest neighbors in such an interval they should be $x_{3}=e^{i \frac{5}{6} \pi}$ and $x_{4}=e^{i \frac{7}{6} \pi}$.

Lemma 3.2. Let $(X, V) \in \mathcal{A C}$. Then $\operatorname{Per}(f) \geq 4$ for every $f \in \mathrm{F}(X, V)$.

Proof. Assume by contradiction that there exists a face $\bar{f} \in \mathrm{F}(X, V)$ having perimeter equal to 3. By definition, $\bar{f}$ is an equilateral triangle with unitary side-length; in particular, denoting by $x_{1}, x_{2}, x_{3}$ the vertices of $\bar{f}$ and by $\hat{x}_{j}(j=1,2,3)$ the inner angles of $\bar{f}$, we have that $\hat{x}_{j}=\frac{\pi}{3}$ for every $j=1,2,3$. 
By the very definition of $\operatorname{Ed}(X, V)$ we have that $\left\langle v\left(x_{j}\right), v\left(x_{k}\right)\right\rangle \geq 0$ for every $j \neq k$. Since, by Lemma 3.1(i), $v\left(x_{j}\right)$ is parallel to the bisector of $\hat{x}_{j}$ for every $j=1,2,3$, we get a contradiction.

Lemma 3.3. Let $(X, V) \in \mathcal{A C}$ and let $f \in \mathrm{F}(X, V)$. If $\operatorname{Per}(f)=4$ then $f$ is a rhombus with side-length equal to one and inner angles equal to $\frac{\pi}{3}, \frac{2}{3} \pi$. In such a case, the orientations of the vertices of $f$ are all equal to each other. Moreover, such an orientation is orthogonal to the shortest diagonal of the rhombus and hence parallel to the longest one.

Proof. Since $f$ is a rhombus, the sum of two consecutive angles equals to $\pi$; hence, by Lemma 3.1(i), we have that the inner angles of $f$ should be equal to $\frac{\pi}{3}, \frac{2}{3} \pi$.

Moreover, by Lemma 3.1(ii) we deduce that all the orientations should be parallel to the bisectors of the $\frac{\pi}{3}$ angles, that in turns are parallel to the longest diagonal of $f$. Finally, since by the very definition of $\operatorname{Ed}(X, V)$, the scalar product between any pair of such orientations should be non-negative, we get that they are all equal to each other.

In view of Lemma 3.3, it is reasonable to denote by $\mathrm{F}^{\diamond}(X, V)$ the set of faces $f$ with $\operatorname{Per}(f)=4$. Note that in this case also $\operatorname{Per}_{\mathrm{gr}}(f)=4$. Moreover, again by Lemma 3.3, we can define the orientation $v(f)$ of a face $f \in \mathrm{F}^{\diamond}(X, V)$ as the orientation of its vertices.

3.2. Geometric decomposition of the energy. Here we decompose the energy $\mathcal{E}$ in $(2.1)$ into the sum of a volume term (which actually depends only on the number of points of the configuration) and a perimeter type term.

Proposition 3.4. For every $(X, V) \in \mathcal{A C}$, it holds

$$
\mathcal{E}(X, V)=-2 \sharp X+\frac{1}{2} \operatorname{def}_{\mathrm{gr}}(X, V)+\frac{1}{2} \operatorname{Per}_{\mathrm{gr}}(X, V)+2 \chi(X, V),
$$

where we have set $\operatorname{def}_{\mathrm{gr}}(X, V):=\sum_{f \in \mathrm{F}^{\mathrm{bad}}(X, V)}\left(\operatorname{Per}_{\mathrm{gr}}(f)-4\right)$ and

$$
\mathrm{F}^{\text {bad }}(X, V):=\mathrm{F}(X, V) \backslash \mathrm{F}^{\diamond}(X, V) .
$$

Proof. The proof relies on the strategy of [9, Theorem 2.1], with relevant differences due to the rhombic lattice we are dealing with. By triangulating each of the faces in $\mathrm{F}^{\text {bad }}(X, V)$ as in Lemma 1.3, one can construct a new planar graph $\overline{\mathrm{G}}(X, V)=(\mathrm{X}, \overline{\mathrm{Ed}}(X, V))$, all of whose faces are either triangles or rhombuses (the latter with unitary side-length). We denote by $\overline{\mathrm{F}}(X, V)$ the set of the faces of $\overline{\mathrm{G}}(X, V)$. Therefore,

$$
\overline{\mathrm{F}}(X, V)=\mathrm{F}^{\diamond}(X, V) \cup \overline{\mathrm{F}}^{\triangle}(X, V),
$$

where $\overline{\mathrm{F}}^{\triangle}(X, V)$ denotes the set of triangular faces of $\overline{\mathrm{G}}(X, V)$. Note that, in view of Lemma $3.2, \overline{\mathrm{F}}^{\triangle}(X, V)$ accounts only for the triangles obtained by triangulating the faces in $\mathrm{F}^{\text {bad }}(X, V)$. Moreover, we denote by $\operatorname{Add}(X, V):=\overline{\mathrm{Ed}}(X, V) \backslash \mathrm{Ed}(X, V)$ the set of the additional edges due to triangulation and by $\overline{\mathrm{Ed}}^{\mathrm{int}}(X, V)$ the set of the interior edges of $\overline{\mathrm{G}}(X, V)$; thus, in view 
of Lemma 1.3, we have

$$
\begin{aligned}
& \overline{\mathrm{Ed}}^{\mathrm{int}}(X, V)=\mathrm{Ed}^{\mathrm{int}}(X, V) \cup \mathrm{Ed}^{\text {wire,int }}(X, V) \cup \operatorname{Add}(X, V) ; \\
& \sharp \operatorname{Add}(X, V)=\sum_{f \in \mathrm{F}^{\mathrm{bad}}(X, V)}\left(\operatorname{Per}_{\mathrm{gr}}(f)-3\right) ; \\
& \sharp \overline{\mathrm{F}}^{\triangle}(X, V)=\sum_{f \in \mathrm{F}^{\mathrm{bad}}(X, V)}\left(\operatorname{Per}_{\mathrm{gr}}(f)-2\right) .
\end{aligned}
$$

By construction, the set of the boundary edges and the set of the exterior wire edges do not change under the triangulation procedure, and hence the graph perimeter of $\mathrm{G}(X, V)$ coincide with the graph perimeter $\overline{\mathrm{G}}(X, V)$. Moreover, the same holds for the Euler characteristic of $\mathrm{G}(X, V)$ in the sense of definition (1.2), i.e., $\chi(\overline{\mathrm{G}}(X, V))=\chi(X, V)$. Notice also that, by the classification of the edges in Section 1, we have

$$
\sharp \mathrm{Ed}^{\text {int }}(X, V)+\sharp \mathrm{Ed}^{\text {wire,int }}(X, V)=\sharp \mathrm{Ed}(X, V)-\sharp \mathrm{Ed}^{\text {wire,ext }}(X, V)-\sharp \mathrm{Ed}^{\partial}(X, V) \text {. }
$$

We note that every exterior wire edge in $\overline{\mathrm{G}}(X, V)$ participates to no face, every boundary edge participates to one face, and all other edges participate to two faces. As a consequence, using (3.3), (3.4) and (3.5), we thus find

$$
\begin{aligned}
& 4 \sharp \mathrm{F}^{\diamond}(X, V)+3 \sharp \overline{\mathrm{F}}^{\triangle}(X, V) \\
= & \sum_{f \in \mathrm{F}^{\diamond}(X, V)} \operatorname{Per}_{\mathrm{gr}}(f)+\sum_{f \in \overline{\mathrm{F}}^{\triangle}(X, V)} \operatorname{Per}_{\mathrm{gr}}(f)=2 \sharp \overline{\mathrm{Ed}}^{\operatorname{int}}(X, V)+\sharp \mathrm{Ed}^{\partial}(X, V) \\
= & 2 \sharp \mathrm{Ed} \mathrm{int}^{\operatorname{int}}(X, V)+2 \sharp \mathrm{Ed}^{\text {wire,int }}(X, V)+2 \sharp \operatorname{Add}(X, V)+\sharp \mathrm{Ed}^{\partial}(X, V) \\
= & 2 \sharp \mathrm{Ed}(X, V)-2 \sharp \mathrm{Ed}^{\text {wire,ext }}(X, V)-\sharp \mathrm{Ed}^{\partial}(X, V)+2 \sharp \operatorname{Add}(X, V) .
\end{aligned}
$$

Moreover, by (3.4), we get

$$
2 \sharp \operatorname{Add}(X, V)-\sharp \overline{\mathrm{F}}^{\triangle}(X, V)=\sum_{f \in \mathrm{F}^{\text {bad }}(X, V)}\left(\operatorname{Per}_{\mathrm{gr}}(f)-4\right)=\operatorname{def}_{\mathrm{gr}}(X, V) .
$$

By (3.6) and (3.7), we obtain

$$
\begin{aligned}
4 \sharp \overline{\mathrm{F}}(X, V)= & 4 \sharp \mathrm{F}^{\diamond}(X, V)+3 \sharp \overline{\mathrm{F}}^{\triangle}(X, V)+\sharp \overline{\mathrm{F}}^{\triangle}(X, V) \\
= & 2 \sharp \mathrm{Ed}(X, V)-2 \sharp \mathrm{Ed}^{\text {wire,ext }}(X, V)-\sharp \mathrm{Ed}^{\partial}(X, V)+2 \sharp \operatorname{Add}(X, V) \\
& +2 \sharp \operatorname{Add}(X, V)-\operatorname{def}_{\mathrm{gr}}(X, V) \\
= & 2 \sharp \mathrm{Ed}(X, V)-\operatorname{Per}_{\mathrm{gr}}(X, V)-\operatorname{def}_{\mathrm{gr}}(X, V)+4 \sharp \operatorname{Add}(X, V),
\end{aligned}
$$

where the last equality follows by (2.4). By (1.2) and (3.8) we deduce that

$$
\begin{aligned}
4 \chi(X, V)= & 4 \sharp \mathrm{X}-4 \sharp \overline{\mathrm{Ed}}(X, V)+4 \sharp \overline{\mathrm{F}}(X, V) \\
= & 4 \sharp \mathrm{X}-4 \sharp \mathrm{Ed}(X, V)-4 \sharp \operatorname{Add}(X, V) \\
& +2 \sharp \mathrm{Ed}(X, V)-\operatorname{Per}_{\mathrm{gr}}(X, V)-\operatorname{def}_{\mathrm{gr}}(X, V)+4 \sharp \operatorname{Add}(X, V) \\
= & 4 \sharp X-2 \sharp \mathrm{Ed}(X, V)-\operatorname{Per}_{\mathrm{gr}}(X, V)-\operatorname{def}_{\mathrm{gr}}(X, V),
\end{aligned}
$$

which yields

$$
-\sharp \operatorname{Ed}(X, V)=-2 \sharp X+\frac{1}{2} \operatorname{def}_{\mathrm{gr}}(X, V)+\frac{1}{2} \operatorname{Per}_{\mathrm{gr}}(X, V)+2 \chi(X, V),
$$


whence the claim follows since, by definition, $\mathcal{E}(X, V)=-\sharp \mathrm{Ed}(X, V)$.

From now on, for every $(X, V) \in \mathcal{A C}$, we set

$$
\mathcal{F}(X, V):=\frac{1}{2} \operatorname{def}_{\mathrm{gr}}(X, V)+\frac{1}{2} \operatorname{Per}_{\mathrm{gr}}(X, V)+2 \chi(X, V),
$$

so that by (3.2), we get $\mathcal{E}(X, V)=-2 \sharp \mathrm{X}+\mathcal{F}(X, V)$.

3.3. Diamond formations. Here we provide rhombic minimizers of the energy $\mathcal{E}$ in $\mathcal{A C}_{N}$ for every $N \in \mathbb{N}$; more precisely, for every $N \in \mathbb{N}$ we construct a minimizing configuration $\left(Y_{N}, W_{N}\right)$ in $\mathcal{A C}_{N}$, referred to as canonical configuration, such that $\mathrm{Y}_{N}$ is a subset of the triangular lattice $\mathcal{T}^{1}$ defined in $(2.5)$ and $\mathrm{F}^{\text {bad }}\left(Y_{N}, W_{N}\right)=\emptyset$.

In order to define $\left(Y_{N}, W_{N}\right)$, we preliminarily note that for every $N \in \mathbb{N}$ there exists a unique pair $(l ; \eta) \in(\mathbb{N} \cup\{0\}) \times(\mathbb{N} \cup\{0\})$ with $0 \leq \eta<2 l+3$ such that

$$
N=(l+1)^{2}+\eta \text {. }
$$

For every $\ell \in \mathbb{N}$, let $R_{\ell}$ be the closed rhombus with inner angles equal to $\frac{\pi}{3}, \frac{2}{3} \pi$, and whose longest diagonal is $\{0\} \times[0, \sqrt{3} \ell]$. Given $l \in \mathbb{N}$, let $\Gamma_{l}:=\partial R_{l+1} \cap\left\{(x, y) \in \mathbb{R}^{2}: y \geq \frac{\sqrt{3}}{2}(l+1)\right\}$. Notice that $\Gamma_{l}$ has length equal to $2 l+2$, and hence it is the support of a curve $\gamma_{l}$ parametrized on $[0,2 l+2]$ and with unitary velocity.

Definition 3.5. We set $\mathrm{Y}_{0}:=\emptyset, \mathrm{Y}_{1}:=\{(0 ; 0)\}, \mathrm{Y}_{2}:=\mathrm{Y}_{1} \cup\left\{\left(\frac{1}{2} ; \frac{\sqrt{3}}{2}\right)\right\}, \mathrm{Y}_{3}:=\mathrm{Y}_{2} \cup\{(0 ; \sqrt{3})\}$. If $N=(l+1)^{2}$ for some $l \in \mathbb{N}$ we define the $\mathrm{Y}_{N}:=R_{l} \cap \mathcal{T}^{1}$, while if $N=(l+1)^{2}+\eta$, for some $l, \eta \in \mathbb{N}$ with $1 \leq \eta \leq 2 l+2$ we set $\mathrm{Y}_{N}:=\left(R_{l} \cap \mathcal{T}^{1}\right) \cup\left\{\gamma_{l}(0)\right\} \ldots \cup\left\{\gamma_{l}(\eta-1)\right\}$. We set $W_{N}:=\{(0 ; 1)\}^{N}$ and we call $\left(Y_{N}, W_{N}\right)$ the canonical configuration in $\mathcal{A C}_{N}$.
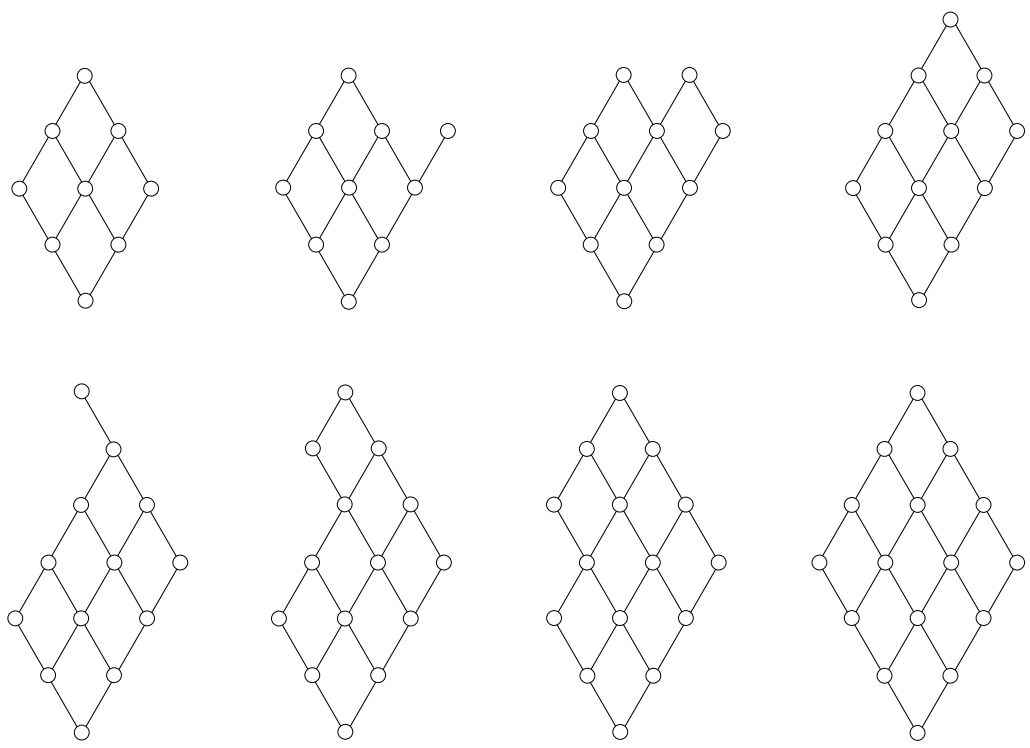

Figure 3. The graphs $\mathrm{G}\left(Y_{N}, W_{N}\right)$ of the configurations $\left(Y_{N}, W_{N}\right)$ for $N=9, \ldots, 16$. 
Note that $\operatorname{Per}_{\mathrm{gr}}\left(Y_{0}, W_{0}\right)=0$ and for every $N \in \mathbb{N}$

$$
\operatorname{Per}_{g r}\left(Y_{N}, W_{N}\right)= \begin{cases}4 l & \text { if } N=(l+1)^{2}, \\ 4 l+2 & \text { if } N=(l+1)^{2}+\eta \text { with } 1 \leq \eta \leq l+1, \\ 4 l+4 & \text { if } N=(l+1)^{2}+\eta \text { with } l+2 \leq \eta \leq 2 l+2 .\end{cases}
$$

Moreover, by construction, $\operatorname{def}_{\mathrm{gr}}\left(Y_{N}, W_{N}\right)=0$ and $\mathrm{G}\left(Y_{N}, W_{N}\right)$ is connected, so that

$$
\mathcal{F}\left(Y_{N}, W_{N}\right)=\frac{1}{2} \operatorname{Per}_{\mathrm{gr}}\left(Y_{N}, W_{N}\right)+2 .
$$

Now, we fix some notations that will be useful to prove that the configurations $\left(Y_{N}, W_{N}\right)$ are minimizers. We recall that $\partial X$ is defined according to Definition 2.1 for $\gamma=\frac{\sqrt{3}}{2}$.

Definition 3.6. For every $N \in \mathbb{N}$ and for every $(X, V) \in \mathcal{A C}_{N}$, we set $N^{\prime}:=N-\sharp \partial X$, $\mathrm{X}^{\prime}:=\mathrm{X} \backslash \partial X$ and we denote by $\left(X^{\prime}, V^{\prime}\right)$ the configuration in $\mathcal{A C}_{N^{\prime}}$ satisfying the following property: for every $j=1, \ldots, N^{\prime}$ there exists a unique $k \in\{1, \ldots, N\}$ such that $x_{j}^{\prime}=x_{k}$, $v_{j}^{\prime}=v_{k}, x_{k} \notin \partial X$.

Notice that, for every $N, N^{\prime}, P \in \mathbb{N} \cup\{0\}$ with $N>N^{\prime}$ there exists a unique pair $(k ; \delta) \in$ $(\mathbb{N} \cup\{0\}) \times(\mathbb{N} \cup\{0\})$ with $\delta<P+8(k+1)$ such that

$$
\begin{aligned}
N & =N^{\prime}+(P+8)+\ldots+(P+8 k)+\delta \\
& =N^{\prime}+k(P+4(k+1))+\delta .
\end{aligned}
$$

We are now in a position to state and prove the main result of this section; the proof relies on some auxiliary lemmas provided in Appendix A.

Theorem 3.7. For every $N \in \mathbb{N}$, it holds

$$
\mathcal{E}\left(Y_{N}, W_{N}\right)=\min _{(X, V) \in \mathcal{A} \mathcal{C}_{N}} \mathcal{E}(X, V) .
$$

Proof. The result is true for $N \in\{1, \ldots, 7\}$ by Lemma A.2. Let $\bar{N} \geq 7$. We will proceed by induction on $N$ and assume that $\left(Y_{N}, W_{N}\right)$ is a minimizer of $\mathcal{F}$ in $\mathcal{A C}_{N}$ for every $N=1, \ldots, \bar{N}$. Let now $N=\bar{N}+1(\geq 8)$ and let $(X, V)$ be a minimizer of $\mathcal{F}$ in $\mathcal{A C}_{N}$. In view of (3.2) we have that $\mathcal{E}(X, V)=-2 \sharp X+\mathcal{F}(X, V)$, where $\mathcal{F}$ is defined in (3.9). It follows that $(X, V)$ minimizes $\mathcal{F}$ in $\mathcal{A C}_{N}$.

By Lemma A.1 and by the inductive assumption, we have that $\mathrm{G}(X, V)$ is connected so that by Lemma 1.2 we have

$$
\begin{aligned}
\mathcal{F}(X, V) & =\frac{1}{2} \operatorname{Per}_{\mathrm{gr}}(X, V)+\frac{1}{2} \operatorname{def}_{\mathrm{gr}}(X, V)+2 \\
& =\frac{1}{2} \sharp \partial X+\frac{1}{2}\left(\operatorname{Per}_{\mathrm{gr}}(X, V)-\sharp \partial X\right)+\frac{1}{2} \operatorname{def}_{\mathrm{gr}}(X, V)+2 \geq \frac{1}{2} \sharp \partial X+2,
\end{aligned}
$$

where the last inequality follows by Lemma A.4 and by the fact that $\operatorname{def}_{\mathrm{gr}}(X, V) \geq 0$. Let $\left(X^{\prime}, V^{\prime}\right)$ be defined as in Definition 3.6. Then, $\left(X^{\prime}, V^{\prime}\right) \in \mathcal{A C}_{N^{\prime}}$ with $N^{\prime}=N-\sharp \partial X<N$. Now, in view of (3.12) applied with $P=\operatorname{Per}_{\text {gr }}\left(Y_{N^{\prime}}, W_{N^{\prime}}\right)$, there exists a unique pair $(k ; \delta) \in$ $(\mathbb{N} \cup\{0\}) \times(\mathbb{N} \cup\{0\})$ with $\delta<\operatorname{Per}_{\operatorname{gr}}\left(Y_{N^{\prime}}, W_{N^{\prime}}\right)+8(k+1)$ such that

$$
\sharp \partial X=N-N^{\prime}=k\left(\operatorname{Per}_{\mathrm{gr}}\left(Y_{N^{\prime}}, W_{N^{\prime}}\right)+4(k+1)\right)+\delta .
$$

We consider three different cases. 
Case 1: $k \geq 2$. By (3.14) and (3.15) we have

$$
\mathcal{F}(X, V) \geq \frac{1}{2} \sharp \partial X+2=\frac{1}{2}\left(k\left(\operatorname{Per}_{g_{r}}\left(Y_{N^{\prime}}, W_{N^{\prime}}\right)+4(k+1)\right)+\delta\right)+2 .
$$

By Lemma A.3, since $k \geq 2$, we have

$$
\operatorname{Per}_{\mathrm{gr}}\left(Y_{N}, W_{N}\right) \leq \operatorname{Per}_{\mathrm{gr}}\left(Y_{N^{\prime}}, W_{N^{\prime}}\right)+8(k+1)
$$

so that, using again that $k \geq 2$, we obtain

$$
k\left(\operatorname{Per}_{\mathrm{gr}}\left(Y_{N^{\prime}}, W_{N^{\prime}}\right)+4(k+1)\right)+\delta \geq \operatorname{Per}_{\mathrm{gr}}\left(Y_{N^{\prime}}, W_{N^{\prime}}\right)+8(k+1) \geq \operatorname{Per}_{\mathrm{gr}}\left(Y_{N}, W_{N}\right)
$$

in view of $(3.16),(3.17)$, and (3.11), we deduce that $\mathcal{F}(X, V) \geq \mathcal{F}\left(Y_{N}, W_{N}\right)$ which implies (3.13).

Case 2: $k=1$. By (3.14), (3.15), Lemma A.3 and (3.11), we have

$$
\begin{aligned}
\mathcal{F}(X, V) & \geq \frac{1}{2} \sharp \partial X+2=\frac{1}{2}\left(\operatorname{Per}_{\mathrm{gr}}\left(Y_{N^{\prime}}, W_{N^{\prime}}\right)+8+\delta\right)+2 \\
& \geq \frac{1}{2}\left(\operatorname{Per}_{\mathrm{gr}}\left(Y_{N^{\prime}}, W_{N^{\prime}}\right)+8+r_{N^{\prime}, N}\right)+2-\frac{1}{2} \\
& =\frac{1}{2} \operatorname{Per}_{\mathrm{gr}}\left(Y_{N}, W_{N}\right)+2-\frac{1}{2}=\mathcal{F}\left(Y_{N}, W_{N}\right)-\frac{1}{2} .
\end{aligned}
$$

Since $\mathcal{F}$ takes values in $\mathbb{N}$, by (3.18) we deduce (3.13) also in this case.

Case 3: $k=0$. By (3.11) and by Lemma A.3 we have

$$
\mathcal{F}\left(Y_{N}, W_{N}\right)=\frac{1}{2} \operatorname{Per}_{\mathrm{gr}}\left(Y_{N}, W_{N}\right)+2 \leq \frac{1}{2}\left(\operatorname{Per}_{\mathrm{gr}}\left(Y_{N^{\prime}}, W_{N^{\prime}}\right)+8\right)+2=\mathcal{F}\left(Y_{N^{\prime}}, W_{N^{\prime}}\right)+4 ;
$$

therefore, by Lemma A.5 and by the inductive assumption, we obtain

$$
\mathcal{F}(X, V) \geq \mathcal{F}\left(X^{\prime}, V^{\prime}\right)+4 \geq \mathcal{F}\left(Y_{N^{\prime}}, W_{N^{\prime}}\right)+4 \geq \mathcal{F}\left(Y_{N}, W_{N}\right)
$$

which by minimality of $(X, V)$ implies (3.13) also in this case.

Remark 3.8. A general rhombic crystallization result for all the minimizers does not hold. Indeed, for all $N$ of the form $N=(l+1)^{2}+1$ or $N=l(l+1)+1$ with $l \geq 1$, one can construct a minimizer having a face $\bar{f}$ with $\operatorname{Per}_{\text {gr }}(\bar{f})=5$, as shown in Figure 4 below. Notice also that for such minimizers the orientation is not constant.

3.4. Compactness of quasi-minimizers. This section is devoted to a compactness result for quasi-minimizers of $\mathcal{E}=\mathcal{E}^{\frac{\sqrt{3}}{2}}$. We start by considering the class of empirical measures associated to the configurations in $\mathcal{A C}$. To this purpose, let $\mathcal{M}$ denote the class of vectorial Radon measures from $\mathbb{R}^{2}$ to $\mathbb{R}^{2}$; for every $N \in \mathbb{N}$ we set

$$
\mathcal{E M}_{N}:=\left\{\mu \in \mathcal{M}: \mu=\sum_{i=1}^{N} v_{i} \delta_{\frac{x_{i}}{\sqrt{N}}} \text { for some }(X, V) \in \mathcal{A C}_{N}\right\} \text {. }
$$

Fix $N \in \mathbb{N}$. Since there is a natural one-to-one correspondence $\mathcal{I}_{N}$ from $\mathcal{E} \mathcal{M}_{N}$ to the class $\mathcal{A C}_{N}$, we can re-define the energy (2.1) on $\mathcal{M}$ introducing the functional $\mathcal{E}_{N}: \mathcal{M} \rightarrow(-\infty,+\infty]$ given by

$$
\mathcal{E}_{N}(\mu):= \begin{cases}\mathcal{E}\left(\mathcal{I}_{N}(\mu)\right) & \text { if } \mu \in \mathcal{E} \mathcal{M}_{N} \\ +\infty & \text { otherwise }\end{cases}
$$



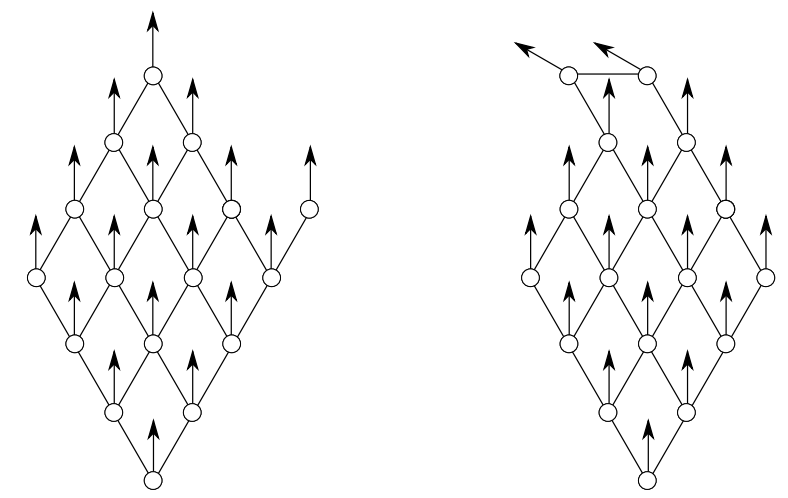

Figure 4. Two minimizers of $\mathcal{E}$ in $\mathcal{A C}_{17}$. On the left: the canonical minimizer. On the right: A minimizer having a non-rhombic face.

In the following, with a little abuse of notation, for every $\mu \in \mathcal{E} \mathcal{M}_{N}$ we will write all the objects introduced in Subsection 2.2 in terms of $\mu$, namely, we set $\operatorname{Ed}(\mu):=\operatorname{Ed}\left(\mathcal{I}_{N}(\mu)\right)$, $\mathrm{F}(\mu):=\mathrm{F}\left(\mathcal{I}_{N}(\mu)\right)$ and so forth. In all this subsection, the symbol Per ${ }^{\text {eu }}$ will denote the De Giorgi's perimeter; recalling the notation introduced in (2.4), (1.4) and (1.5), we have

$$
\begin{aligned}
\operatorname{Per}(\mu) & =\operatorname{Per}\left(\mathcal{I}_{N}(\mu)\right)=\operatorname{Per}^{\mathrm{eu}}(O(\mu)), \\
\operatorname{Per}_{\mathrm{gr}}(\mu) & =\operatorname{Per}_{\mathrm{gr}}\left(\mathcal{I}_{N}(\mu)\right)=\operatorname{Per}^{\mathrm{eu}}(O(\mu))+2 \sharp \operatorname{Ed}^{\text {wire,ext }}(\mu), \\
\operatorname{Per}_{\mathrm{gr}}(f) & =\operatorname{Per}^{\mathrm{eu}}(f)+2 \sharp \mathrm{Ed}^{\text {wire,int }}(f) \quad \text { for every face } f \in \mathrm{F}(\mu) .
\end{aligned}
$$

Moreover, setting

we have

$$
f_{N}:=\frac{f}{\sqrt{N}} \quad \text { for every } f \in \mathrm{F}(\mu)
$$

$$
\operatorname{Per}^{\mathrm{eu}}\left(f_{N}\right)=\frac{\operatorname{Per}^{\mathrm{eu}}(f)}{\sqrt{N}} \quad \text { for every } f \in \mathrm{F}(\mu)
$$

so that

$$
\operatorname{Per}^{\mathrm{eu}}\left(f_{N}\right) \geq \frac{5}{\sqrt{N}} \quad \text { for every } f \in \mathrm{F}^{\mathrm{bad}}(\mu) \text {; }
$$

furthermore, setting $O_{N}(\mu):=\frac{1}{\sqrt{N}} O(\mu)=\bigcup_{f \in \mathrm{F}(\mu)} f_{N}$, it holds

$$
\operatorname{Per}^{\mathrm{eu}}\left(O_{N}(\mu)\right)=\frac{\operatorname{Per}^{\mathrm{eu}}(O(\mu))}{\sqrt{N}} .
$$

Therefore, by (3.2) and (3.19), the functionals $\mathcal{E}_{N}$ can be decomposed

$$
\begin{aligned}
\mathcal{E}_{N}(\mu)+2 \mu\left(\mathbb{R}^{2}\right)= & \frac{\sqrt{N}}{2} \sum_{f \in \mathrm{Fbad}^{\mathrm{ba}}(\mu)}\left(\operatorname{Per}^{\mathrm{eu}}\left(f_{N}\right)-\frac{4}{\sqrt{N}}\right) \\
& +\frac{\sqrt{N}}{2} \operatorname{Per}^{\mathrm{eu}}\left(O_{N}(\mu)\right)+\sharp \mathrm{Ed}^{\text {wire }}(\mu)+2 \chi(\mu) .
\end{aligned}
$$

We can finally state the desired compactness result. 
Theorem 3.9. Let $\left\{\mu_{N}\right\} \subset \mathcal{M}$ be such that

$$
\mathcal{E}_{N}\left(\mu_{N}\right)+2 \mu_{N}\left(\mathbb{R}^{2}\right) \leq C \sqrt{N} \text { for some } C \in \mathbb{R} .
$$

Then, up to a subsequence, $\frac{\sqrt{3}}{2 N} \mu_{N} \stackrel{*}{\rightarrow} \mu$ for some $\mu \in \mathcal{M}$. Moreover, $\mu=\sum_{j \in J} v_{j} \mathbb{1}_{\omega_{j}} \mathrm{~d} x$ where $J \subseteq \mathbb{N}, v_{j} \in \mathcal{S}^{1}$ for every $j \in J$, and $\sum_{j \in J} \operatorname{Per}^{\mathrm{eu}}\left(\omega_{j}\right)<+\infty$.

Before providing the proof of Theorem 3.9 we state the following compactness property which is a corollary of Ambrosio compactness result [1] and of [2, Theorem 4.23].

Proposition 3.10. Let $\left\{u_{h}\right\} \subset S B V\left(\mathbb{R}^{2} ; \mathbb{R}^{2}\right)$. If there exists $p>1$ and $C>0$ such that

$$
\int_{\mathbb{R}^{2}}\left|\nabla u_{h}\right|^{p} \mathrm{~d} x+\mathcal{H}^{1}\left(S_{u_{h}}\right)+\left\|u_{h}\right\|_{L^{\infty}\left(\mathbb{R}^{2}\right)} \leq C \text { for all } h \in \mathbb{N}
$$

then, up to a subsequence, there exists $u \in S B V\left(\mathbb{R}^{2}\right)$ such that for every open bounded set $A \subset \mathbb{R}^{2}$ it holds

$$
\begin{aligned}
& u_{h} \rightarrow u \text { in } L^{1}\left(A ; \mathbb{R}^{2}\right), \\
& \nabla u_{h} \rightarrow \nabla u \text { in } L^{1}\left(A ; \mathbb{R}^{2 \times 2}\right), \\
& \liminf _{h \rightarrow \infty} \mathcal{H}^{1}\left(S_{u_{h}} \cap A\right) \geq \mathcal{H}^{1}\left(S_{u} \cap A\right) .
\end{aligned}
$$

Moreover, if $\nabla u=0$, then $u=\sum_{j \in J} v_{j} \mathbb{1}_{\omega_{j}} \mathrm{~d} x$ where $J \subseteq \mathbb{N}, v_{j} \in \mathbb{R}^{2}$ for every $j \in J$, and $\sum_{j \in J} \operatorname{Per}^{\mathrm{eu}}\left(\omega_{j}\right)<+\infty$.

In the following we say that a sequence $\left\{u_{h}\right\}_{h}$ converges to some function $u$ in $S B V_{\text {loc }}\left(\mathbb{R}^{2} ; \mathbb{R}^{2}\right)$ if $u_{h} \rightarrow u$ in $L_{\text {loc }}^{1}\left(\mathbb{R}^{2} ; \mathbb{R}^{2}\right)$ and $\left\{u_{h}\right\}_{h}$ satisfies (3.23) for some $p>1$.

Proof of Theorem 3.9. The proof is divided into two steps.

Step 1. Let $\left\{\rho_{N}\right\}_{N \in \mathbb{N}}$ be the sequence defined by

$$
\rho_{N}:=\sum_{f \in \mathrm{F}^{\diamond}\left(\mu_{N}\right)} v(f) \mathbb{1}_{f_{N}} \quad \text { for every } N \in \mathbb{N}
$$

we first claim that, up to a subsequence, $\rho_{N} \rightarrow \rho$ in $S B V_{\text {loc }}\left(\mathbb{R}^{2} ; \mathbb{R}^{2}\right)$ for some function $\rho$ of the form $\rho:=\sum_{j \in J} v_{j} \mathbb{1}_{\omega_{j}}$ where $v_{j}$ and $\omega_{j}$ satisfy the claim of the theorem.

In view of (3.22), (3.21), (3.20), and (1.3), we have

$$
\begin{aligned}
C & \geq \frac{1}{\sqrt{N}}\left(\mathcal{E}_{N}\left(\mu_{N}\right)+2 \mu_{N}\left(\mathbb{R}^{2}\right)\right) \geq \frac{1}{2} \operatorname{Per}^{\mathrm{eu}}\left(O_{N}\left(\mu_{N}\right)\right)+\frac{1}{10} \sum_{f \in \mathrm{Fbad}^{\mathrm{bad}}\left(\mu_{N}\right)} \operatorname{Per}^{\mathrm{eu}}\left(f_{N}\right) \\
& \geq \frac{1}{10} \operatorname{Per}^{\mathrm{eu}}\left(\bigcup_{f \in \mathrm{F}^{\diamond}\left(\mu_{N}\right)} f_{N}\right)=\frac{1}{10} \operatorname{Per}^{\mathrm{eu}}\left(\Omega_{N}\left(\mu_{N}\right)\right),
\end{aligned}
$$

where we have denoted by $\Omega_{N}\left(\mu_{N}\right)$ the interior of $\bigcup_{f \in \mathrm{F} \diamond\left(\mu_{N}\right)} \operatorname{clos}\left(f_{N}\right)$. By the very definition of $\rho_{N}$ we have that $\left\|\rho_{N}\right\|_{L^{\infty}} \leq 1, \nabla \rho_{N}=0$, and $\mathcal{H}^{1}\left(S_{\rho_{N}}\right) \leq \operatorname{Per}^{\mathrm{eu}}\left(\Omega_{N}\left(\mu_{N}\right)\right) \leq 10 C$.

Then, the claim directly follows by Proposition 3.10 .

Step 2. Now we prove that, up to a subsequence,

$$
\frac{\sqrt{3}}{2 N} \mu_{N} \stackrel{*}{\rightarrow} \rho \mathrm{d} x \quad \text { as } N \rightarrow+\infty
$$


with $\rho$ provided by Step 1 . To this purpose, for every $f \in \mathrm{F}^{\diamond}\left(\mu_{N}\right)$ we denote by $a_{j}\left(f_{N}\right)$ $(j=1,2,3,4)$ the vertices of $f_{N}$, and we define

$$
\hat{\mu}_{N}:=\frac{1}{4 N} \sum_{f \in \mathrm{F} \diamond\left(\mu_{N}\right)} v(f) \sum_{j=1}^{4} \delta_{a_{j}\left(f_{N}\right)}, \quad \widetilde{\mu}_{N}:=\rho_{N} \mathrm{~d} x .
$$

By (3.24) and by the isoperimetric inequality, we obtain

$$
\int_{\mathbb{R}^{2}}\left|\rho_{N}\right| \mathrm{d} x=\left|\widetilde{\mu}_{N}\right|\left(\mathbb{R}^{2}\right)=\left|\Omega_{N}\left(\mu_{N}\right)\right| \leq C .
$$

We now show that $\frac{\sqrt{3}}{2} \hat{\mu}_{N}-\widetilde{\mu}_{N} \stackrel{*}{\rightarrow} 0$. Let $\psi \in C_{c}^{0}\left(\mathbb{R}^{2}\right)$; for every $f \in \mathrm{F}^{\diamond}\left(\mu_{N}\right)$, let $\psi_{f_{N}}$ be the average of $\psi$ on $f_{N}$. Then

$$
\begin{aligned}
\left|\left\langle\frac{\sqrt{3}}{2} \hat{\mu}_{N}-\widetilde{\mu}_{N}, \psi\right\rangle\right| & =\mid \sum_{f \in \mathrm{F} \diamond\left(\mu_{N}\right)}\left\langle\frac{\sqrt{3}}{2} \hat{\mu}_{N}-\widetilde{\mu}_{N}, \psi\left\llcorner\operatorname{clos}\left(f_{N}\right)\right\rangle\right| \\
& =\mid \sum_{f \in \mathrm{F} \diamond\left(\mu_{N}\right)}\left\langle\frac{\sqrt{3}}{2} \hat{\mu}_{N}-\widetilde{\mu}_{N},\left(\psi-\psi_{f_{N}}\right)\left\llcorner\operatorname{clos}\left(f_{N}\right)\right\rangle\right| \\
& \leq \sum_{f \in \mathrm{F} \diamond\left(\mu_{N}\right)} \mid\left\langle\frac{\sqrt{3}}{2} \hat{\mu}_{N}-\widetilde{\mu}_{N},\left(\psi-\psi_{f_{N}}\right)\left\llcorner\operatorname{clos}\left(f_{N}\right)\right\rangle\right| \\
& \leq 2\left|\widetilde{\mu}_{N}\right|\left(\mathbb{R}^{2}\right) r_{\psi}\left(\frac{1}{N}\right) \leq C r_{\psi}\left(\frac{1}{N}\right) \rightarrow 0,
\end{aligned}
$$

where $r_{\psi}$ is the modulus of continuity of $\psi$.

Now we prove that $\left|\frac{1}{N} \mu_{N}-\hat{\mu}_{N}\right|\left(\mathbb{R}^{2}\right) \rightarrow 0$ as $N \rightarrow+\infty$. We set $Z_{N}:=\left\{x \in \operatorname{supp} \mu_{N}\right.$ : $x$ does not lie on four rhombic faces $\}$ and we claim that

$$
\sharp Z_{N} \leq c \sqrt{N},
$$

for some positive constant $c \in \mathbb{R}$ (independent of $N$ ).

In order to prove (3.28) we first notice that, in view of Lemma 3.1(iii), $Z_{N}=Z_{N}^{4} \cup Z_{N}^{<4}$, where

$$
\begin{aligned}
Z_{N}^{4} & :=\left\{x \in Z_{N}: x \text { lies on four bonds }\right\} \text { and } \\
Z_{N}^{<4}: & =\left\{x \in Z_{N}: x \text { lies on } k \text { bonds with } k \leq 3\right\} .
\end{aligned}
$$

By (3.21) and (3.22), we have

$$
\frac{1}{4} \sharp Z_{N}^{4} \leq \sqrt{N} \operatorname{Per}^{\mathrm{eu}}\left(O_{N}\left(\mu_{N}\right)\right)+\sharp \mathrm{Ed}^{\text {wire }}\left(\mu_{N}\right)+\sqrt{N} \sum_{f \in \mathrm{F}^{\mathrm{bad}}\left(\mu_{N}\right)} \operatorname{Per}^{\mathrm{eu}}\left(f_{N}\right) \leq \bar{C} \sqrt{N},
$$

which, setting $c_{1}:=4 \bar{C}$, implies that

$$
\sharp Z_{N}^{4} \leq c_{1} \sqrt{N} .
$$

Moreover, again by (3.22) we get

$$
C \sqrt{N} \geq \mathcal{E}_{N}\left(\mu_{N}\right)+2 \mu_{N}\left(\mathbb{R}^{2}\right)=\frac{1}{2} \sum_{x \in \operatorname{supp} \mu_{N}}\left(4-\sharp\left\{e \in \operatorname{Ed}\left(\mu_{N}\right): x \in e\right\}\right) \geq \frac{1}{2} \sharp Z_{N}^{<4},
$$


which, setting $c_{2}:=2 C$, implies that

$$
\sharp Z_{N}^{<4} \leq c_{2} \sqrt{N} .
$$

By summing (3.29) and (3.30) and setting $c:=c_{1}+c_{2}$, we get (3.28). By (3.28), we get

$$
\left|\frac{1}{N} \mu_{N}-\hat{\mu}_{N}\right|\left(\mathbb{R}^{2}\right) \leq \frac{1}{N} \sharp Z_{N} \leq \frac{c}{\sqrt{N}} \rightarrow 0 .
$$

By combining (3.27) and (3.31) we obtain (3.25).

\section{Appendix A. Auxiliary lemmas}

This appendix is devoted to the results used in the proof of Theorem 3.7.

Lemma A.1. Let $\bar{N} \in \mathbb{N}$. If (3.13) holds true for every $N \leq \bar{N}$, then every minimizer $(X, V)$ of $\mathcal{E}$ in $\mathcal{A C}_{\bar{N}+1}$ is such that $\mathrm{G}(X, V)$ is connected.

Proof. Let $(X, V)$ be a minimizer of $\mathcal{F}$ in $\mathcal{A C}_{\bar{N}+1}$. We argue by contradiction: Let $\mathrm{G}_{\mathrm{x}_{1}}, \ldots, \mathrm{G}_{\mathrm{x}_{K}}$ with $K \geq 2$ be the connected components of $\mathrm{G}(X, V)$ according to Definition 1.1 and set $\mathrm{G}_{\mathrm{X}}=\mathrm{G}\left(X_{k}, V_{k}\right)$ and $N_{k}:=\sharp \mathrm{X}_{k}$ for every $k=1, \ldots, K$. Then, $\bar{N}+1=\sum_{k=1}^{K} N_{k}$ and $1 \leq N_{k} \leq \bar{N}$ for every $k=1, \ldots, K$.

By the very definition of $\mathcal{E}$ in $(2.1)$

$$
\mathcal{E}(X, V)=-\sum_{k=1}^{K} \sharp \operatorname{Ed}\left(X_{k}, V_{k}\right)=\sum_{k=1}^{K} \mathcal{E}\left(X_{k}, V_{k}\right) .
$$

By (A.1) and by the assumption (3.13) for $N_{k} \leq \bar{N}$, we deduce

$$
\mathcal{E}(X, V)=\sum_{k=1}^{K} \mathcal{E}\left(X_{k}, V_{k}\right) \geq \sum_{k=1}^{K} \mathcal{E}\left(Y_{N_{k}}, W_{N_{k}}\right) .
$$

Now we will glue all the configurations $\left(Y_{N_{k}}, W_{N_{k}}\right)$ creating new bonds and hence decreasing the energy. To this purpose, for every $k=1, \ldots, K$ and for every $\tau \in \mathbb{R}^{2}$ let $\mathrm{Y}_{N_{k}}(\tau):=\mathrm{Y}_{N_{k}}+\tau$ and let $\left(Y_{N_{k}}(\tau), W_{N_{k}}\right)$ be the corresponding configuration. By construction, $\mathcal{E}\left(Y_{N_{k}}, W_{N_{k}}\right)=$ $\mathcal{E}\left(Y_{N_{k}}(\tau), W_{N_{k}}\right)$. Since $W_{N_{k}}=\{(0 ; 1)\}^{N_{k}}$ for every $k=1, \ldots, K$, it is easy to see that there exist translations $\tau_{1}, \ldots, \tau_{k} \in \mathbb{R}^{2}$ such that the configuration $\left(\bar{Y}_{\bar{N}+1}, \bar{W}_{\bar{N}+1}\right)$ with $\bar{W}_{\bar{N}+1}=$ $\{(0 ; 1)\}^{\bar{N}+1}$ and $\overline{\mathrm{Y}}_{\bar{N}+1}:=\bigcup_{k=1}^{K} \mathrm{Y}_{N_{k}}\left(\tau_{k}\right)$ is in $\mathcal{A C}_{\bar{N}+1}$ and satisfies that $\mathrm{G}\left(\bar{Y}_{\bar{N}+1}, \bar{W}_{\bar{N}+1}\right)$ is connected (so that new bonds have been formed). It follows that

$$
\mathcal{E}\left(\bar{Y}_{\bar{N}+1}, \bar{W}_{\bar{N}+1}\right)<\sum_{k=1}^{K} \mathcal{E}\left(Y_{N_{k}}, W_{N_{k}}\right) \leq \mathcal{E}(X, V),
$$

thus contradicting the minimality of $(X, V)$.

Now we show that (3.13) is satisfied for small number of particles.

Lemma A.2. For every $N \in\{1, \ldots, 7\}$, (3.13) holds true.

Proof. The claim is trivially satisfied for $N=1$ and $N=2$. 
Note that for $N=3$, a minimizer $(X, V)$ of $\mathcal{E}$ in $\mathcal{A C}_{N}$ cannot have three bonds, since such three bonds should form an equilateral triangle with unitary side-length, which contradicts Lemma 3.2. Therefore, we get

$$
-2 \leq \min _{(X, V) \in \mathcal{A C}_{3}} \mathcal{E}(X, V) \leq \mathcal{E}\left(Y_{3}, W_{3}\right)=-2 .
$$

Now we focus on the case $N=4$. Let $(X, V)$ be a minimizer of $\mathcal{E}$ in $\mathcal{A C}_{4}$. By Lemma A.1 below $\mathrm{G}(X, V)$ is connected. Let $P$ be the maximum among the perimeters $\operatorname{Per}_{\mathrm{gr}}(f)$ of the faces $f$ of $(X, V)$. If $P=0$, then $(X, V)$ has no faces and hence by Euler formula $\sharp \mathrm{X}-\sharp \mathrm{Ed}(X, V)=1$ so that

$$
\mathcal{E}(X, V)=-(\sharp X-1)=-3>-4=\mathcal{E}\left(Y_{4}, W_{4}\right),
$$

thus contradicting the minimality of $(X, V)$. It follows that $P \geq 3$ and in fact, by Lemma 3.2 , $P \geq 4$. Therefore, $P=4$ and by Lemma 3.3, $\mathrm{X}$ is the set of the vertices of a rhombus with unitary side-length and $v(x)$ is the same for every $x \in \mathrm{X}$. It follows that $(X, V)$ coincides up to a rigid motion for $\mathbf{X}$ and up to flipping the orientation field $V$ - with $\left(Y_{4}, W_{4}\right)$.

As for $N=5$, let $(X, V)$ be a minimizer of $\mathcal{E}$ in $\mathcal{A C}_{5}$. By Lemma A.1 $\mathrm{G}(X, V)$ is connected and by Lemmas 3.2 and 3.3, $\mathrm{G}(X, V)$ can have at most one face. Moreover, if $(X, V)$ has no faces, i.e., only wires are present, by arguing as in (A.2) we get a contradiction. As a consequence, $(X, V)$ has exactly one face $f$ and either $\operatorname{Per}(f)=5$ and there are no further edges or $\operatorname{Per}(f)=4$ and there is a wire edge. In both cases we have

$$
\mathcal{E}(X, V)=-5=\mathcal{E}\left(Y_{5}, W_{5}\right) .
$$

Now we consider the case $N=6$. Let $(X, V)$ be a minimizer of $\mathcal{E}$ in $\mathcal{A C}_{6}$. By Lemma A.1 $\mathrm{G}(X, V)$ is connected. Since $\left(Y_{6}, W_{6}\right)$ is a competitor for the minimum problem in (3.13), we have

$$
\mathcal{E}(X, V) \leq \mathcal{E}\left(Y_{6}, W_{6}\right)=-7 .
$$

As in the previous cases, we can immediately exclude that $(X, V)$ has no faces. Moreover, $(X, V)$ cannot have only one face; indeed, if there is a unique face then by Euler formula

$$
\mathcal{E}(X, V)=-\sharp \mathrm{X}=-6>-7,
$$

thus contradicting the minimality of $(X, V)$. In view of Lemma 3.2 we have that the only possibility is that $(X, V)$ has exactly two faces $f_{1}$ and $f_{2}$ with $\operatorname{Per}\left(f_{j}\right)=4(j=1,2)$; therefore, $(X, V)$ has no wires and, by Lemma $3.3, f_{1}$ and $f_{2}$ are rhombuses with unitary side-length. Moreover, since $\mathrm{G}(X, V)$ is connected we have that $\partial f_{1}$ and $\partial f_{2}$ share at least one vertex and actually, since $N=6, f_{1}$ and $f_{2}$ share one bond. It follows that $(X, V)$ coincides - up to a rigid motion for $\mathrm{X}$ and up to flipping the orientation field $V$ - with $\left(Y_{6}, W_{6}\right)$.

We conclude by proving (3.13) for $N=7$. Let $(X, V)$ be a minimizer of $\mathcal{E}$ in $\mathcal{A C}_{7}$. By Lemma A.1 $\mathrm{G}(X, V)$ is connected. Since $\left(Y_{7}, W_{7}\right)$ is a competitor for the minimum problem in (3.13), we have

$$
\mathcal{E}(X, V) \leq \mathcal{E}\left(Y_{7}, W_{7}\right)=-8 .
$$

By arguing as in (A.2) and (A.3) we deduce that $(X, V)$ has at least two faces.

Notice that, by the hard sphere condition, it easily follows that if a face $f \in \mathrm{F}(X, V)$ has $\operatorname{Per}(f) \leq 5$, then $f$ is convex and $\operatorname{Per}_{\mathrm{gr}}(f)=\operatorname{Per}(f)$. If a face $f_{1}$ with maximal perimeter has $\operatorname{Per}\left(f_{1}\right)=5$ then, by Lemma 3.2, the only possibility is that $(X, V)$ has no wires and one further face $f_{2}$ such that $\operatorname{Per}\left(f_{2}\right)=4$ and $f_{1}$ and $f_{2}$ share one edge. In such a case, we have 
that $\mathcal{E}(X, V)=-8=\mathcal{E}\left(Y_{7}, W_{7}\right)$. Now, if a face $f_{1}$ with maximal perimeter has $\operatorname{Per}\left(f_{1}\right)=4$ then, by arguing as above we have that there exists one further face $f_{2}$ such that $\operatorname{Per}\left(f_{2}\right)=4$. Since the graph is connected, we can have either that $f_{1}$ and $f_{2}$ share only one vertex and that there are no wires or that $f_{1}$ and $f_{2}$ share one edge and that there is a wire. In both cases we deduce that $\mathcal{E}(X, V)=-8=\mathcal{E}\left(Y_{7}, W_{7}\right)$ which implies the claim also for $N=7$ and concludes the proof of the lemma.

By the very definition of $\left(Y_{N}, W_{N}\right)$ we have the following result.

Lemma A.3. Let $N, N^{\prime} \in \mathbb{N} \cup\{0\}$ with $N>N^{\prime}$. Let $(k ; \delta) \in(\mathbb{N} \cup\{0\}) \times(\mathbb{N} \cup\{0\})$ be the pair provided by (3.12) for $P=\operatorname{Per}_{\mathrm{gr}}\left(Y_{N^{\prime}}, W_{N^{\prime}}\right)$. Then

$$
\operatorname{Per}_{\mathrm{gr}}\left(Y_{N}, W_{N}\right)=\operatorname{Per}_{\mathrm{gr}}\left(Y_{N^{\prime}}, W_{N^{\prime}}\right)+8 k+r_{N^{\prime}, N}
$$

where $r_{N^{\prime}, N} \in\{0,2,4,6,8\}$ and $r_{N^{\prime}, N}=0$ if $\delta=0$. Moreover, if $k \geq 1$, then $r_{N^{\prime}, N} \leq 2\left\lceil\frac{\delta}{2}\right\rceil$.

Proof. We preliminarily note that $r_{N^{\prime}, N}$ is even since $\operatorname{Per}_{\mathrm{gr}}\left(Y_{N}, W_{N}\right)$ is even for every $N \in \mathbb{N}$.

We first prove that if $\delta=0$, then (A.4) holds true with $r_{N^{\prime}, N}=0$, i.e., we show that if $N=N^{\prime}+k\left(\operatorname{Per}_{\mathrm{gr}}\left(Y_{N^{\prime}}, W_{N^{\prime}}\right)+4(k+1)\right)$ for some $k \in \mathbb{N}$, then

$$
\operatorname{Per}_{\mathrm{gr}}\left(Y_{N}, W_{N}\right)=\operatorname{Per}_{\mathrm{gr}}\left(Y_{N^{\prime}}, W_{N^{\prime}}\right)+8 k \text {. }
$$

We proceed by induction on $k$ starting from $k=1$.

Let $k=1$. If $N^{\prime}=0$ then $N=8$ and, by (3.10), we get $\operatorname{Per}_{\mathrm{gr}}\left(Y_{N}, W_{N}\right)=8=$ $\operatorname{Per}_{\text {gr }}\left(Y_{N^{\prime}}, W_{N^{\prime}}\right)+8$. If $N^{\prime} \geq 1$, then $N^{\prime}=(l+1)^{2}+\eta$ for some $l, \eta \in \mathbb{N} \cup\{0\}$ with $0 \leq \eta \leq 2 l+2$. We prove (A.5) (with $k=1$ ) only for $\eta=0$, the other cases being fully analogous. Since $\eta=0$, we have $N^{\prime}=(l+1)^{2}$ for some $l \in \mathbb{N} \cup\{0\}$; then, by (3.10), $\operatorname{Per}_{\mathrm{gr}}\left(Y_{N^{\prime}}, W_{N^{\prime}}\right)=4 l$ so that

$$
N=N^{\prime}+\operatorname{Per}_{\mathrm{gr}}\left(Y_{N^{\prime}}, W_{N^{\prime}}\right)+8=(l+1)^{2}+4 l+8=(l+3)^{2},
$$

which, in view of (3.10) yields

$$
\operatorname{Per}_{\mathrm{gr}}\left(Y_{N}, W_{N}\right)=4(l+2)=4 l+8=\operatorname{Per}_{\mathrm{gr}}\left(Y_{N^{\prime}}, W_{N^{\prime}}\right)+8,
$$

i.e., (A.5).

Assume now that (A.5) holds true for every $k=1, \ldots, \hat{k}$, and let us show that it is satisfied also for $k=\hat{k}+1$. Let $N^{\prime} \in \mathbb{N} \cup\{0\}, k=\hat{k}+1$ and, in turn, $N=N^{\prime}+(\hat{k}+1)\left(\operatorname{Per}_{\mathrm{gr}}\left(Y_{N^{\prime}}, W_{N^{\prime}}\right)+\right.$ $4(\hat{k}+2))$. We set $\bar{N}^{\prime}:=N^{\prime}+\hat{k}\left(\operatorname{Per}_{\mathrm{gr}}\left(Y_{N^{\prime}}, W_{N^{\prime}}\right)+4(\hat{k}+1)\right)$; applying the inductive step to $N^{\prime}, k=\hat{k}$ and $N=\bar{N}^{\prime}$ we have

$$
\operatorname{Per}_{\mathrm{gr}}\left(Y_{\bar{N}^{\prime}}, W_{\bar{N}^{\prime}}\right)=\operatorname{Per}_{\mathrm{gr}}\left(Y_{N^{\prime}}, W_{N^{\prime}}\right)+8 \hat{k} .
$$

It follows that

$$
\begin{aligned}
N & =N^{\prime}+(\hat{k}+1)\left(\operatorname{Per}_{\mathrm{gr}}\left(Y_{N^{\prime}}, W_{N^{\prime}}\right)+4(\hat{k}+2)\right) \\
& =N^{\prime}+\hat{k}\left(\operatorname{Per}_{\mathrm{gr}}\left(Y_{N^{\prime}}, W_{N^{\prime}}\right)+4(\hat{k}+1)\right)+4 \hat{k}+\operatorname{Per}_{\mathrm{gr}}\left(Y_{N^{\prime}}, W_{N^{\prime}}\right)+4(\hat{k}+2) \\
& =\bar{N}^{\prime}+\operatorname{Per}_{\mathrm{gr}}\left(Y_{N^{\prime}}, W_{N^{\prime}}\right)+8 \hat{k}+8=\bar{N}^{\prime}+\operatorname{Per}_{\mathrm{gr}}\left(Y_{\bar{N}^{\prime}}, W_{\bar{N}^{\prime}}\right)+8 .
\end{aligned}
$$

By the step $k=1$ applied to $N^{\prime}$ replaced by $\bar{N}^{\prime}$ and, in turn, $N=N$ and by (A.6) we get

$$
\operatorname{Per}_{\mathrm{gr}}\left(Y_{N}, W_{N}\right)=\operatorname{Per}_{\mathrm{gr}}\left(Y_{\bar{N}^{\prime}}, W_{\bar{N}^{\prime}}\right)+8=\operatorname{Per}_{\mathrm{gr}}\left(Y_{N^{\prime}}, W_{N^{\prime}}\right)+8(\hat{k}+1)
$$

thus implying the claim and concluding the proof of (A.4) for $\delta=0$ with $r_{N^{\prime}, N}=0$. 
Now we prove (A.4) in the general case. Let $N=N^{\prime}+k\left(\operatorname{Per}_{\mathrm{gr}}\left(Y_{N^{\prime}}, W_{N^{\prime}}\right)+4(k+1)\right)+\delta$ with $\delta<\operatorname{Per}_{\text {gr }}\left(Y_{N^{\prime}}, W_{N^{\prime}}\right)+8(k+1)$. We set $N_{1}:=N^{\prime}+k\left(\operatorname{Per}_{\text {gr }}\left(Y_{N^{\prime}}, W_{N^{\prime}}\right)+4(k+1)\right)$ and $N_{2}:=N^{\prime}+(k+1)\left(\operatorname{Per}_{\mathrm{gr}}\left(Y_{N^{\prime}}, W_{N^{\prime}}\right)+4(k+2)\right)$ we have that $N_{1} \leq N<N_{2}$; by applying (A.5) with $N$ replaced by $N_{1}$ and $N_{2}$ respectively and using the monotonicity of $\operatorname{Per}_{\mathrm{gr}}\left(Y_{N}, W_{N}\right)$ with respect to $N$ we obtain

$$
\begin{aligned}
\operatorname{Per}_{\mathrm{gr}}\left(Y_{N^{\prime}}, W_{N^{\prime}}\right)+8 k & =\operatorname{Per}_{\mathrm{gr}}\left(Y_{N_{1}}, W_{N_{1}}\right) \\
& \leq \operatorname{Per}_{\mathrm{gr}}\left(Y_{N}, W_{N}\right) \leq \operatorname{Per}_{\mathrm{gr}}\left(Y_{N_{2}}, W_{N_{2}}\right)=\operatorname{Per}_{\mathrm{gr}}\left(Y_{N^{\prime}}, W_{N^{\prime}}\right)+8(k+1),
\end{aligned}
$$

which concludes the proof of (A.4).

Finally we show that $r_{N^{\prime}, N} \leq 2\left\lceil\frac{\delta}{2}\right\rceil$ if $k \geq 1$. Let $N^{\prime} \in \mathbb{N} \cup\{0\}$ and let $k \geq 1$. Let $N:=N^{\prime}+k\left(\operatorname{Per}_{\mathrm{gr}}\left(Y_{N^{\prime}}, W_{N^{\prime}}\right)+4(k+1)\right)+\delta$ and set

$$
\widetilde{N}:=N^{\prime}+k\left(\operatorname{Per}_{g_{r}}\left(Y_{N^{\prime}}, W_{N^{\prime}}\right)+4(k+1)\right)
$$

so that $N=\tilde{N}+\delta$. Since we are assuming that $k \geq 1$, we have that $\tilde{N} \geq 8$. By (A.4) and by applying (A.5) with $N^{\prime}=N^{\prime}, k=k$ and $N=\widetilde{N}$ we have

$$
\operatorname{Per}_{\mathrm{gr}}\left(Y_{N}, W_{N}\right)=\operatorname{Per}_{\mathrm{gr}}\left(Y_{N^{\prime}}, W_{N^{\prime}}\right)+8 k+r_{N^{\prime}, N}=\operatorname{Per}_{\mathrm{gr}}\left(Y_{\widetilde{N}}, W_{\widetilde{N}}\right)+r_{N^{\prime}, N} .
$$

For every $\delta \in \mathbb{N} \cup\{0\}$ we set

$$
p(\delta):=\operatorname{Per}_{\mathrm{gr}}\left(Y_{\widetilde{N}+\delta}, W_{\widetilde{N}+\delta}\right)-\operatorname{Per}_{\mathrm{gr}}\left(Y_{\widetilde{N}}, W_{\widetilde{N}}\right) .
$$

Note that $p(\delta) \in 2 \mathbb{N} \cup\{0\}$ for every $\delta \in \mathbb{N} \cup\{0\}$ and $p(0)=0$. We denote by $J_{p}:=\left\{\delta_{i}\right\} \subseteq \mathbb{N}$ with $\delta_{i}<\delta_{i+1}$ the set of the "jump discontinuities" of $p$, i.e., such that $p\left(\delta_{i}\right)>p\left(\delta_{i}-1\right)$. Since $\widetilde{N} \geq 8$, in view of $(3.10)$, for every $\delta_{i} \in J_{p}$ we have

$$
p\left(\delta_{i}\right)-p\left(\delta_{i}-1\right)=2, \quad\left|\delta_{i+1}-\delta_{i}\right| \geq 3 .
$$

This fact together with $p(0)=0$ yields $p(\delta) \leq 2\left\lceil\frac{\delta}{2}\right\rceil$ for every $\delta \in \mathbb{N}$ and hence in view of (A.7) and (A.8) we obtain that $r_{N^{\prime}, N}=p(\delta) \leq 2\left\lceil\frac{\delta}{2}\right\rceil$ for every $\delta \in \mathbb{N}$.

Lemma A.4. Let $N \in \mathbb{N}$ with $N \geq 2$ and let $(X, V) \in \mathcal{A C}_{N}$ be such that $\mathrm{G}(X, V)$ is connected. Then

$$
\operatorname{Per}_{\mathrm{gr}}(X, V) \geq \sharp \partial X .
$$

Proof. Set $\widetilde{X}:=\partial X, \widetilde{\mathrm{Ed}}:=\operatorname{Ed}^{\partial}(X, V) \cup \operatorname{Ed}^{\text {wire,ext }}(X, V)$ and $\widetilde{\mathrm{G}}=(\widetilde{\mathrm{X}}, \widetilde{\mathrm{Ed}})$. According to Section $1, F(\widetilde{G})$ denotes the set of faces of $\widetilde{G}$.

Since $\mathrm{G}(X, V)$ is connected, we have that $\widetilde{\mathrm{G}}$ is connected too, so that by Lemma 1.2 we have

$$
\sharp \widetilde{X}-\sharp \widetilde{E d}+\sharp F(\widetilde{G})=1,
$$

i.e.,

$$
\sharp \partial X=1-\sharp \mathrm{F}(\widetilde{\mathrm{G}})+\sharp \mathrm{Ed}^{\partial}(X, V)+\sharp \mathrm{Ed}^{\text {wire,ext }}(X, V) .
$$

If $\sharp \mathrm{F}(\widetilde{\mathrm{G}})=0$, then $\sharp \mathrm{Ed}^{\partial}(X, V)=0$ and, since $N \geq 2$ and $\mathrm{G}(X, V)$ is connected, we have $\sharp \mathrm{Ed}^{\text {wire,ext }}(X, V) \geq 1$, so that

$$
\sharp \partial X=1+\sharp \mathrm{Ed}^{\text {wire,ext }}(X, V) \leq 2 \sharp \mathrm{Ed}^{\text {wire,ext }}(X, V)=\operatorname{Per}_{\mathrm{gr}}(X, V) .
$$


If $\sharp \mathrm{F}(\widetilde{\mathrm{G}}) \geq 1$, by $(\mathrm{A} .10)$ we get

$$
\sharp \partial X \leq \sharp \mathrm{Ed}^{\partial}(X, V)+\sharp \mathrm{Ed}^{\text {wire,ext }}(X, V) \leq \sharp \mathrm{Ed}^{\partial}(X, V)+2 \sharp \mathrm{Ed}^{\text {wire,ext }}(X, V)=\operatorname{Per}_{\mathrm{gr}}(X, V) \text {. }
$$

In both cases we have proven (A.9).

Lemma A.5. Let $N \in \mathbb{N}$ with $N \geq 8$. Let $(X, V) \in \mathcal{A C}_{N}$ with $\mathrm{G}(X, V)$ connected. Then

$$
\mathcal{F}(X, V) \geq \mathcal{F}\left(X^{\prime}, V^{\prime}\right)+4,
$$

where $\left(X^{\prime}, V^{\prime}\right)$ is as in Definition 3.6.

Before proving Lemma A.5, we need some auxiliary lemmas. We start by fixing some notations.

Let $N \geq 8$ and let $(X, V) \in \mathcal{A C}_{N}$ be such that $\mathrm{G}(X, V)$ is connected, $O(X, V)$ has simple and closed polygonal boundary and $\sharp \mathrm{Ed}^{\text {wire,ext }}(X, V)=0$. For every $x \in \partial X$, we set

$$
\bar{\imath}(x):=\sharp\left\{e \in \operatorname{Ed}^{\operatorname{int}}(X, V): x \in e\right\} .
$$

Moreover, for every $x \in \partial X$ we denote by $\hat{x}$ the inner angle spanned by the two boundary edges containing $x$. Here by inner angle we mean the angle that is "interior" to $O(X, V)$. In view of Lemma 3.1 we can classify the points $x \in \partial X$ in the following subclasses:

$$
\begin{aligned}
Y^{j}:=\left\{x \in \partial X: \hat{x}=j \frac{\pi}{3}\right\}, & j=1, \ldots, 5, \\
Y^{j, j+1}:=\left\{x \in \partial X: \hat{x} \in\left(j \frac{\pi}{3},(j+1) \frac{\pi}{3}\right)\right\}, & j=2,3,
\end{aligned}
$$

i.e.,

$$
\partial X=\bigcup_{j=1}^{5} Y^{j} \cup Y^{2,3} \cup Y^{3,4} .
$$

By Lemma 3.1(iii) every point in $\mathrm{X}$ can lie on at most four bonds, so that $\bar{\imath}(x) \leq 2$ for every $x \in \partial X$. Therefore, for every $k=0,1,2$ we can set

$$
\begin{aligned}
Y_{k}^{j} & :=\left\{x \in Y^{j}: \bar{\imath}(x)=k\right\} \quad \text { for } j=1,2,3,4,5, \\
Y_{k}^{j, j+1} & :=\left\{x \in Y^{j, j+1}: \bar{\imath}(x)=k\right\} \quad \text { for } j=2,3 .
\end{aligned}
$$

By Lemma 3.1 we have

$$
\begin{aligned}
Y^{1}=Y_{0}^{1}, \quad Y^{2}=Y_{0}^{2}, \quad Y^{2,3}=Y_{0}^{2,3}, \quad Y^{3}=Y_{0}^{3} \cup Y_{1}^{3} \\
Y^{3,4}=Y_{0}^{3,4} \cup Y_{1}^{3,4}, \quad Y^{4}=Y_{0}^{4} \cup Y_{1}^{4} \cup Y_{2}^{4}, \quad Y^{5}=Y_{0}^{5} \cup Y_{1}^{5} \cup Y_{2}^{5} .
\end{aligned}
$$

Definition A.6. Given two boundary particles $x^{\prime}, x^{\prime \prime} \in Y^{1} \cup Y^{2} \cup Y^{2,3} \cup Y^{4} \cup Y^{5}=\partial X \backslash$ $\left(Y^{3} \cup Y^{3,4}\right)$ we say that $x^{\prime}$ follows $x^{\prime \prime}$ and we write $x^{\prime} \rightarrow x^{\prime \prime}$ if there exist $M \in \mathbb{N}$ and a path $x^{\prime}=z_{0}, \ldots, z_{M}=x^{\prime \prime}$ in $\partial X$, oriented according to the counter-clockwise orientation of $\partial O(X, V)$, such that $\left\{z_{m-1}, z_{m}\right\} \in \operatorname{Ed}^{\partial}(X, V)$ and $z_{m} \in Y^{3} \cup Y^{3,4}$ for every $m=1, \ldots, M-1$.

Note that the case $M=1$ in Definition A.6 corresponds to $\left\{x^{\prime}, x^{\prime \prime}\right\} \in \operatorname{Ed}^{\partial}(X, V)$. Notice also that for every point $x^{\prime} \in \partial X \backslash\left(Y^{3} \cup Y^{3,4}\right)$ there exists a unique point $x^{\prime \prime} \in \partial X \backslash\left(Y^{3} \cup Y^{3,4}\right)$ such that $x^{\prime} \rightarrow x^{\prime \prime}$. Moreover, we set

$$
Y_{k}^{j} \rightarrow Y_{a}^{b}:=\left\{x^{\prime} \in Y_{k}^{j}: x^{\prime} \rightarrow x^{\prime \prime} \text { for some } x^{\prime \prime} \in Y_{a}^{b}\right\},
$$

and $Y_{k}^{j} \rightarrow\left\{Y_{a_{1}}^{b_{1}}, \ldots, Y_{a_{L}}^{b_{L}}\right\}:=\bigcup_{l=1}^{L} Y_{k}^{j} \rightarrow Y_{a_{l}}^{b_{l}}$. 
For every $x \in \partial X$ and for every $z_{1}, z_{2} \in \mathrm{X}$ with $\left\{x, z_{1}\right\}\left\{x, z_{2}\right\} \in \operatorname{Ed}(X, V)$, we denote by $\widehat{z_{1} x z_{2}}$ the angle formed by $\left[x, z_{1}\right]$ and $\left[x, z_{2}\right]$ that is "interior" to $O(X, V)$. Notice that, if also $z_{1}, z_{2} \in \partial X$, then $\left\{x, z_{1}\right\}\left\{x, z_{2}\right\} \in \mathrm{Ed}^{\partial}(X, V)$ and $\hat{x}=\widehat{z_{1} x z_{2}}$.

As a consequence of Lemma 3.1, we have the following result.

Lemma A.7. Let $(X, V) \in \mathcal{A C}$ be such that $\mathrm{G}(X, V)$ is connected, $O(X, V)$ has simple and closed polygonal boundary and $\mathrm{Ed}^{\text {wire,ext }}(X, V)=\emptyset$. The following facts hold true.

(i) If $x, y, z_{1}, z_{2} \in \mathrm{X}$ are such that $x \in Y_{1}^{3} \cup Y_{1}^{3,4},\left\{x, z_{1}\right\},\left\{x, z_{2}\right\} \in \operatorname{Ed}^{\partial}(X, V)$ and $\{x, y\} \in \operatorname{Ed}^{\text {int }}(X, V)$, then either $\widehat{z_{1} x y}=\frac{\pi}{3}$ or $\widehat{y x z_{2}}=\frac{\pi}{3}$. Moreover, $v(x)$ is parallel to the bisector of the $\frac{\pi}{3}$ angle.

(ii) If $x, y_{1}, y_{2}, z_{1}, z_{2} \in \mathrm{X}$ are such that $x \in Y_{2}^{4},\left\{x, z_{1}\right\},\left\{x, z_{2}\right\} \in \mathrm{Ed}^{\partial}(X, V)$, $\left\{x, y_{1}\right\},\left\{x, y_{2}\right\} \in \operatorname{Ed}^{\text {int }}(X, V)$ and $z_{1}, z_{2}, y_{2}, y_{1}$ are counter-clockwise oriented, then $\widehat{z_{1} x y_{1}}=\frac{\pi}{3}, \widehat{y_{1} x y_{2}}=\frac{2}{3} \pi$ and $\widehat{y_{2} x z_{2}}=\frac{\pi}{3}$. Moreover, $v(x)$ is parallel to the bisector of the angles $\widehat{z_{1} x y_{1}}$ and $\widehat{y_{2} x z_{2}}$.

(iii) If $x, y_{1}, y_{2}, z_{1}, z_{2} \in \mathrm{X}$ are such that $x \in Y_{2}^{5},\left\{x, z_{1}\right\},\left\{x, z_{2}\right\} \in \mathrm{Ed}^{\partial}(X, V)$, $\left\{x, y_{1}\right\},\left\{x, y_{2}\right\} \in \operatorname{Ed}^{\mathrm{int}}(X, V)$ and and $z_{1}, z_{2}, y_{2}, y_{1}$ are counter-clockwise oriented, then $\widehat{z_{1} x y_{1}}=\frac{2}{3} \pi, \widehat{y_{1} x y_{2}}=\frac{\pi}{3}$ and $\widehat{y_{2} x z_{2}}=\frac{2}{3} \pi$. Moreover, $v(x)$ is parallel to the bisector of the angle $\widehat{y_{1} x y_{2}}$.

Lemma A.8. Let $(X, V) \in \mathcal{A C}$ be such that $\mathrm{G}(X, V)$ is connected, $O(X, V)$ has simple and closed polygonal boundary and $\mathrm{Ed}^{\text {wire,ext }}(X, V)=\emptyset$. Let $x, y \in \partial X$.

If $x, y \in Y_{0}^{1}$ and $x \rightarrow y$, then $M \geq 2$ in Definition A.6 and there exists $z_{m}$ as in Definition A.6 such that $z_{m} \in Y_{0}^{3} \cup Y_{0}^{3,4}$.

The same statement holds true also if $x, y \in Y_{2}^{4}$ or if $x \in Y_{0}^{1}, y \in Y_{2}^{4}$ or if $x \in Y_{2}^{4}, y \in Y_{0}^{1}$.

Proof. We prove the claim only for $x, y \in Y_{0}^{1}$, being the proof in the other cases fully analogous. By Lemma 3.2, we immediately get $M \geq 2$. Assume by contradiction that $z_{m} \in Y_{1}^{3} \cup Y_{1}^{3,4}$ for every $m=1, \ldots, M-1$. For every $m=1, \ldots, M-1$ let $w_{m}$ be the only point in $\mathrm{X}$ such that $\left\{z_{m}, w_{m}\right\} \in \mathrm{Ed}^{\text {int }}(X, V)$. By Lemma 3.2 we have that the points $w_{m}$ are all distinct. Moreover, by Lemma 3.2 and by Lemma A.7(i) we get $\widehat{w_{1} z_{1} z_{2}}=\frac{\pi}{3}$; analogously, it follows that $\widehat{w_{2} z_{2} z_{3}}=\frac{\pi}{3}$ and in fact, by induction, that $w_{m} \widehat{z_{m} z_{m}+1}=\frac{\pi}{3}$ for every $m=1, \ldots, M-1$. In particular, $w_{M-1} \widehat{z_{M}-1} z_{M}=\frac{\pi}{3}$, thus contradicting Lemma 3.2.

Proof of Lemma A.5. We preliminarily prove the claim under the assumptions that $O(X, V)$ has simple and closed polygonal boundary and $\sharp \mathrm{Ed}^{\text {wire,ext }}(X, V)=0$; in such a case

$$
\operatorname{Per}_{\mathrm{gr}}(X, V)=\operatorname{Per}(X, V)=\sharp \partial X .
$$

Set

$$
I:=\left\{e \in \operatorname{Ed}^{\text {int }}(X, V): \exists x \in \partial X \text { such that } x \in e\right\} ;
$$

recalling (A.11), we have

$$
\sharp I \leq \sum_{x \in \partial X} \bar{\imath}(x) .
$$

In view of $(2.1),(3.2)$, and (3.9), we have

$$
\begin{aligned}
-\operatorname{Per}(X, V)-\sharp I=\mathcal{E}(X, V)-\mathcal{E}\left(X^{\prime}, V^{\prime}\right) & =-2 \sharp \mathrm{X}+\mathcal{F}(X, V)+2 \sharp \mathrm{X}^{\prime}-\mathcal{F}\left(X^{\prime}, V^{\prime}\right) \\
& =-2 \sharp \partial X+\mathcal{F}(X, V)-\mathcal{F}\left(X^{\prime}, V^{\prime}\right),
\end{aligned}
$$


whence, together with (A.15) and (A.16), we deduce

$$
\mathcal{F}(X, V)-\mathcal{F}\left(X^{\prime}, V^{\prime}\right)=\sharp \partial X-\sharp I \geq \sum_{x \in \partial X}(1-\bar{\imath}(x)) .
$$

Therefore, in order to prove the claim it is enough to show that

$$
\sum_{x \in \partial X}(1-\bar{\imath}(x)) \geq 4
$$

Recalling (A.12) and (A.13) we have

$$
\begin{aligned}
\sum_{x \in \partial X}(1-\bar{\imath}(x))= & \sharp Y_{0}^{1}-\sharp Y_{2}^{5}+\sharp Y_{0}^{2}-\sharp Y_{2}^{4} \\
& +\sharp Y_{0}^{2,3}+\sharp Y_{0}^{3}+\sharp Y_{0}^{3,4}+\sharp Y_{0}^{4}+\sharp Y_{0}^{5} .
\end{aligned}
$$

Since $O(X, V)$ is connected, the Gauss-Bonnet formula gives $2 \pi=\sum_{x \in \partial X}(\pi-\hat{x})$, where $\hat{x}$ denotes the angle at $x$ interior to $O(X, V)$; therefore,

$$
2 \pi=\sum_{x \in \partial X}(\pi-\hat{x}) \leq \frac{2}{3} \pi \sharp Y^{1}+\frac{\pi}{3} \sharp Y^{2}+\frac{\pi}{3} \sharp Y^{2,3}-\frac{\pi}{3} \sharp Y^{4}-\frac{2}{3} \pi \sharp Y^{5},
$$

which, in view of (A.13), yields

$$
6 \leq 2\left(\sharp Y_{0}^{1}-\sharp Y_{2}^{5}\right)+\sharp Y_{0}^{2}-\sharp Y_{2}^{4}-r
$$

where

$$
r:=2 \sharp Y_{1}^{5}+2 \sharp Y_{0}^{5}+\sharp Y_{1}^{4}+\sharp Y_{0}^{4}-\sharp Y_{0}^{2,3} .
$$

We point out that we are making no claims on the on the sign of $r$.

Recalling the notation introduced in (A.14), for every $(j ; k) \in\{(1 ; 0),(2 ; 0),(4 ; 2),(5 ; 2)\}$, we set

$$
A_{k}^{j}:=Y_{k}^{j} \rightarrow\left\{Y_{0}^{2}, Y_{2}^{5}\right\}, \quad B_{k}^{j}:=Y_{k}^{j} \rightarrow\left\{Y_{0}^{1}, Y_{2}^{4}\right\}, \quad C_{k}^{j}:=Y_{k}^{j} \rightarrow\left\{Y_{0}^{2,3}, Y_{0}^{4}, Y_{1}^{4}, Y_{0}^{5}, Y_{1}^{5}\right\},
$$

so that

$$
\sharp Y_{k}^{j}=\sharp A_{k}^{j}+\sharp B_{k}^{j}+\sharp C_{k}^{j} \quad \text { for every }(j ; k) \in\{(1 ; 0),(2 ; 0),(4 ; 2),(5 ; 2)\} .
$$

By construction

$$
\begin{gathered}
\sharp A_{0}^{1}+\sharp A_{0}^{2}+\sharp A_{2}^{4}+\sharp A_{2}^{5} \leq \sharp Y_{0}^{2}+\sharp Y_{2}^{5}, \\
\sharp C_{0}^{1}+\sharp C_{0}^{2}+\sharp C_{2}^{4}+\sharp C_{2}^{5} \leq \sharp Y_{0}^{2,3}+\sharp Y_{0}^{4}+\sharp Y_{1}^{4}+\sharp Y_{0}^{5}+\sharp Y_{1}^{5},
\end{gathered}
$$

and, by Lemma A.8,

$$
\sharp B_{0}^{1}+\sharp B_{2}^{4} \leq \sharp Y_{0}^{3}+\sharp Y_{0}^{3,4} \text {. }
$$

By applying (A.21) with $(j ; k)=(1 ; 0)$ and $(j ; k)=(4 ; 2)$, summing such identities, and using (A.22), we have

$$
\begin{aligned}
\sharp Y_{0}^{1}+\sharp Y_{2}^{4}-\left(\sharp B_{0}^{1}+\sharp C_{0}^{1}+\sharp B_{2}^{4}+\sharp C_{2}^{4}\right) & =\sharp A_{0}^{1}+\sharp A_{2}^{4} \\
& \leq \sharp A_{0}^{1}+\sharp A_{2}^{4}+\sharp A_{0}^{2}+\sharp A_{2}^{5} \leq \sharp Y_{0}^{2}+\sharp Y_{2}^{5} .
\end{aligned}
$$


Moreover, in view of (A.23) and (A.24), we have

$$
\begin{aligned}
& -\left(\sharp B_{0}^{1}+\sharp C_{0}^{1}+\sharp B_{2}^{4}+\sharp C_{2}^{4}\right) \\
\geq & -\left(\sharp B_{0}^{1}+\sharp B_{2}^{4}+\sharp C_{0}^{1}+\sharp C_{0}^{2}+\sharp C_{2}^{4}+\sharp C_{2}^{5}\right) \\
\geq & -\sharp Y_{0}^{2,3}-\sharp Y_{0}^{4}-\sharp Y_{1}^{4}-\sharp Y_{0}^{5}-\sharp Y_{1}^{5}-\sharp Y_{0}^{3}-\sharp Y_{0}^{3,4}=: \bar{\delta},
\end{aligned}
$$

which, by (A.25), yields

$$
\sharp Y_{0}^{1}+\sharp Y_{2}^{4}+\bar{\delta} \leq \sharp Y_{0}^{2}+\sharp Y_{2}^{5} .
$$

Therefore

$$
\sharp Y_{0}^{2}+\sharp Y_{2}^{5}=\sharp Y_{0}^{1}+\sharp Y_{2}^{4}+\delta \quad \text { for some } \delta \geq \bar{\delta},
$$

or, equivalently,

$$
\sharp Y_{0}^{2}-\sharp Y_{2}^{4}=\sharp Y_{0}^{1}-\sharp Y_{2}^{5}+\delta \quad \text { for some } \delta \geq \bar{\delta} \text {. }
$$

By (A.27) and by (A.19), we deduce that

$$
6 \leq 2\left(\sharp Y_{0}^{1}-\sharp Y_{2}^{5}\right)+\sharp Y_{0}^{1}-\sharp Y_{2}^{5}+\delta-r
$$

so that

$$
\sharp Y_{0}^{1}-\sharp Y_{2}^{5} \geq 2+\frac{r}{3}-\frac{\delta}{3},
$$

and hence, by (A.27), we get

$$
\sharp Y_{0}^{2}-\sharp Y_{2}^{4} \geq 2+\frac{r}{3}+\frac{2}{3} \delta .
$$

By (A.18), (A.28), (A.29), (A.20) and (A.26) we obtain

$$
\begin{aligned}
\sum_{x \in \partial X}(1-\bar{\imath}(x)) \geq & 4+\frac{2}{3} r+\frac{\bar{\delta}}{3}+\sharp Y_{0}^{2,3}+\sharp Y_{0}^{3}+\sharp Y_{0}^{3,4}+\sharp Y_{0}^{4}+\sharp Y_{0}^{5} \\
\geq & 4+\frac{4}{3} \sharp Y_{1}^{5}+\frac{4}{3} \sharp Y_{0}^{5}+\frac{2}{3} \sharp Y_{1}^{4}+\frac{2}{3} \sharp Y_{0}^{4}-\frac{2}{3} \sharp Y_{0}^{2,3} \\
& -\frac{1}{3} \sharp Y_{0}^{2,3}-\frac{1}{3} \sharp Y_{0}^{4}-\frac{1}{3} \sharp Y_{1}^{4}-\frac{1}{3} \sharp Y_{0}^{5}-\frac{1}{3} \sharp Y_{1}^{5}-\frac{1}{3} \sharp Y_{0}^{3}-\frac{1}{3} \sharp Y_{0}^{3,4} \\
& +\sharp Y_{0}^{2,3}+\sharp Y_{0}^{3}+\sharp Y_{0}^{3,4}+\sharp Y_{0}^{4}+\sharp Y_{0}^{5} \\
= & 4+\frac{2}{3} \sharp Y_{0}^{3}+\frac{2}{3} \sharp Y_{0}^{3,4}+\frac{4}{3} \sharp Y_{0}^{4}+2 \sharp Y_{0}^{5}+\frac{1}{3} \sharp Y_{1}^{4}+\sharp Y_{1}^{5} \\
\geq & 4,
\end{aligned}
$$

which implies (A.17) and hence the claim in the case that $O(X, V)$ has simple and closed polygonal boundary and $\sharp \mathrm{Ed}^{\text {wire,ext }}(X, V)=0$.

If $O(X, V)$ is connected and $\operatorname{Ed}^{\text {wire,ext }}(X, V)=\emptyset$ (without assuming that $O(X, V)$ has simple and closed polygonal boundary), we argue in the following manner. By Lemma 3.1(iii), every point $x \in \partial X$ can lie either on two boundary edges or on four boundary edges. We set

$$
\mathrm{X}_{\text {bow }}:=\{x \in \partial X: x \text { lies on four boundary edges }\} \text {. }
$$

Let $\hat{O}_{1}(X, V), \ldots, \hat{O}_{K}(X, V)$ be the connected components of $O(X, V) \backslash \mathrm{X}_{\text {bow }}$. For every $k=1, \ldots, K$ we set $\widetilde{O}_{k}(X, V):=\operatorname{clos}\left(\hat{O}_{k}(X, V)\right), \widetilde{\mathrm{X}}_{k}:=\mathrm{X} \cap \widetilde{O}_{k}(X, V)$ and we denote by $\left(\widetilde{X}_{k}, \widetilde{V}_{k}\right)$ the corresponding configuration. Clearly, $O\left(\widetilde{X}_{k}, \widetilde{V}_{k}\right)=\widetilde{O}_{k}(X, V)$ has simple and 
closed polygonal boundary, $\mathrm{G}\left(\widetilde{X}_{k}, \widetilde{V}_{k}\right)$ is connected and Ed ${ }^{\text {wire,ext }}\left(\widetilde{X}_{k}, \widetilde{V}_{k}\right)=\emptyset$; therefore, by the above proven result, we get

$$
\mathcal{F}\left(\widetilde{X}_{k}, \widetilde{V}_{k}\right) \geq \mathcal{F}\left(\tilde{X}_{k}^{\prime}, \widetilde{V}_{k}^{\prime}\right)+4 \quad \text { for every } k=1, \ldots, K .
$$

Since $\sum_{k=1}^{K} \mathcal{F}\left(\widetilde{X}_{k}^{\prime}, \widetilde{V}_{k}^{\prime}\right)=\mathcal{F}\left(X^{\prime}, V^{\prime}\right)$, by (A.30) we get

$$
\begin{aligned}
\mathcal{F}(X, V) & =\frac{1}{2} \operatorname{Per}_{\mathrm{gr}}(X, V)+\frac{1}{2} \operatorname{def}_{\mathrm{gr}}(X, V)+2 \\
& =\sum_{k=1}^{K}\left(\frac{1}{2} \operatorname{Per}_{\mathrm{gr}}\left(\widetilde{X}_{k}, \widetilde{V}_{k}\right)+\frac{1}{2} \operatorname{def}_{\mathrm{gr}}\left(\widetilde{X}_{k}, \widetilde{V}_{k}\right)\right)+2 \\
& =\sum_{k=1}^{K} \mathcal{F}\left(\widetilde{X}_{k}, \widetilde{V}_{k}\right)+2(1-K) \geq \sum_{k=1}^{K}\left(\mathcal{F}\left(\widetilde{X}_{k}^{\prime}, \widetilde{V}_{k}^{\prime}\right)+4\right)+2(1-K) \\
& =\mathcal{F}\left(X^{\prime}, V^{\prime}\right)+2 K+2 \geq \mathcal{F}\left(X^{\prime}, V^{\prime}\right)+4 .
\end{aligned}
$$

Finally we treat the general case. Let $O_{1}(X, V), \ldots, O_{K}(X, V)$ be the connected components of $O(X, V)$. For every $k=1, \ldots, K$ we set $\mathrm{X}_{k}:=\mathrm{X} \cap O_{k}(X, V)$ and we denote by $\left(X_{k}, V_{k}\right)$ the corresponding configuration. Clearly, $O\left(X_{k}, V_{k}\right)=O_{k}(X, V)$ and $\mathrm{Ed}^{\text {wire,ext }}\left(X_{k}, V_{k}\right)=\emptyset$, so that, by (A.31), we have

$$
\mathcal{F}\left(X_{k}, V_{k}\right) \geq \mathcal{F}\left(X_{k}^{\prime}, V_{k}^{\prime}\right)+4 \quad \text { for every } k=1, \ldots, K .
$$

Now, since $\sum_{k=1}^{K} \mathcal{F}\left(X_{k}^{\prime}, V_{k}^{\prime}\right)=\mathcal{F}\left(X^{\prime}, V^{\prime}\right)$, by (A.32) we obtain

$$
\begin{aligned}
\mathcal{F}(X, V) & =\frac{1}{2} \operatorname{Per}_{\mathrm{gr}}(X, V)+\frac{1}{2} \operatorname{def}_{\mathrm{gr}}(X, V)+2 \geq \sum_{k=1}^{K}\left(\frac{1}{2} \operatorname{Per}_{\mathrm{gr}}\left(X_{k}, V_{k}\right)+\frac{1}{2} \operatorname{def}_{\mathrm{gr}}\left(X_{k}, V_{k}\right)\right)+2 \\
& =\sum_{k=1}^{K} \mathcal{F}\left(X_{k}, V_{k}\right)+2(1-K) \geq \sum_{k=1}^{K}\left(\mathcal{F}\left(X_{k}^{\prime}, V_{k}^{\prime}\right)+4\right)+2(1-K) \geq \mathcal{F}\left(X^{\prime}, V^{\prime}\right)+4,
\end{aligned}
$$

which concludes the proof of the Lemma.

\section{REFERENCES}

[1] L. Ambrosio: Existence theory for a new class of variational problems, Arch. Ration. Mech. Anal. 111 (1990), 291-322.

[2] L. Ambrosio, N. Fusco, D. Pallara: Functions of Bounded Variation and Free Discontinuity Problems, Oxford University Press, Oxford, 2000.

[3] Y. Au Yeung, G. Friesecke, B. Schmidt: Minimizing atomic configurations of short range pair potentials in two dimensions: crystallization in the Wulff shape, Calc. Var. Partial Differ. Equ. 44 (2012), 81-100.

[4] M Ballerini, N. Cabibbo, R. Candelier, A. Cavagna, E. Cisbani, I. Giardina, V. Lecomte, A. Orlandi, G. Parisi, A. Procaccini, M. Viale, V. Zdravkovic: Interaction ruling animal collective behavior depends on topological rather than metric distance: Evidence from a field study, Proc. Natl. Acad. Sci. U.S.A. 105 (2008), 1232-1237.

[5] L. Bétermin, L. De Luca, M. Petrache: Crystallization to the square lattice for a two-body potential, preprint available at http://cvgmt.sns.it/media/doc/paper/4502/BeDePe_final.pdf .

[6] C.M. Breder: Vortices and fish schools, Zoologica 50 (1965), 97-114.

[7] C.M. Breder: Fish schools as operational structures, Fish. Bull. 74 (1976), 471-502.

[8] L. De Luca, G. Friesecke: Classification of particle numbers with unique Heitmann-Radin minimizer, $J$. Stat. Phys. 167 (2017), 1586-1592. 
[9] L. De Luca, G. Friesecke: Crystallization in two dimensions and a discrete Gauss-Bonnet theorem, J. Nonlinear Sci. 28 (2018), 69-90.

[10] L. De Luca, M. Novaga, M. Ponsiglione: $\Gamma$-convergence of the Heitmann-Radin sticky disc energy to the crystalline perimeter, J. Nonlinear Sci. 29 (2019), 1273-1299.

[11] F.E. Fish: Energetics of swimming and flying in formation, Comments Theor. Biol. 5 (1999), $283-304$.

[12] M. Friedrich, L. Kreutz, B. Schmidt: Emergence of rigid Polycrystals from atomistic Systems with Heitmann-Radin sticky disk energy, preprint (2020) available at https://arxiv.org/abs/2006.01558

[13] R. C. Heitmann, C. Radin: The ground state for sticky disks, J. Stat. Phys. 22 (1980), 281-287.

[14] A. Kubin, M. Ponsiglione: Attractive Riesz potentials acting on hard spheres, preprint (2020).

[15] J.C. Liao: A review of fish swimming mechanics and behaviour in altered flows, Phil. Trans. R. Soc. B 362 (2007), 1973-1993.

[16] G.H. Meisters: Polygons have ears, Am. Math. Mon. 82 (1975), 648-651.

[17] E.G. Noya, C. Vega, J.P. Doye, A.A. Louis: The stability of a crystal with diamond structure for patchy particles with tetrahedral symmetry, J. Chem. Phys. 132 (2010), 234511.

[18] J.K. Parrish, L. Edelstein-Keshet: Complexity, pattern, and evolutionary trade-offs in animal aggregation, Science 284 (1999), 99-101.

[19] C.W. Reynolds: Flocks, herds, and schools: A distributed behavioral model, Comp. Graph. 21 (1987), $25-33$.

[20] F. Theil: A proof of crystallization in two dimensions, Commun. Math. Phys. 262 (2006), 209-236.

[21] D. Weihs: Hydromechanics of fish schooling, Nature 241 (1973), 290-291.

(Lucia De Luca) IAC-CNR, Via Dei TAurini, 19 I-00184 Rome, ItAly

E-mail address, L. De Luca: lucia.deluca@cnr.it

(Angelo Ninno) Dipartimento di Matematica "G. Castelnuovo", Sapienza Università di Roma, Piazzale A. Moro 2, I-00185, Rome, Italy

E-mail address, A. Ninno: angelo.ninno@uniroma1.it

(Marcello Ponsiglione) Dipartimento di Matematica "G. Castelnuovo", Sapienza Università di Roma, Piazzale A. Moro 2, I-00185, Rome, Italy

E-mail address, M. Ponsiglione: ponsigli@mat.uniroma1.it 